\title{
Über den Einfluss intravenöser Zufuhr von Säure bzw. Alkali auf das Eiweiss und den kolloid-osmotischen Druck des Blutes bei normalen sowie nierenkranken Kaninchen.
}

\author{
Von \\ Kiyoshi Endo. \\ （遠 藤 清） \\ (Aus der Medizinischen Klinik von Prof. Dr. T. Kato, \\ Tohoku Reichsuniversität zu Sendai.)
}

Dass die Lebenserscheinungen des Organismus durch Säurebasengleichgewichtsstörung im Blute verschiedenerweise beeinflusst werden, ist schon yon vielen Seiten eingehend untersucht und es ist bereits allgemein bekannt, dass Säuren oder Alkalien auf Wasserhaushalt im Organismus bedeutende Einflüsse ausüben.

Loeb ${ }^{1)}$ fand, dass Zusatz einer kleinen Menge von stark verdünnten Säuren oder Basen eine starke Gewichtszunahme eines in physiologischer Kochsalzlösung befindlichen Froschmuskels veranlasst. $\mathrm{Hambuxger^{2 } )}$ konstatierte an Pferden, dass die Menge der festen Bestandteile im Serum nach intravenöser Einspritzung von isotonischer $\mathrm{Na}_{2} \mathrm{SO}_{4}$-Lösung in Mengen von 2 Liter, in welcher sich $40 \mathrm{ccm}$ Schwefelsäure $(20 \mathrm{ccm}$ konzentrierte Schwefelsäure $+20 \mathrm{ccm}$ Wasser) befindet, zunimmt, während sie nach intravenöser Einverleibung von isotonischer $\mathrm{Na}_{2} \mathrm{SO}_{4}$ Lösung in gleicher Menge, welche $0,2 \% \mathrm{NaOH}$ enthält, abnimmt. Pfeiffer ${ }^{3}$, Wyss ${ }^{4)}$, Maase u. Zonde ${ }^{5}$, Jansen ${ }^{6)}$ u. a. haben berichtet, dass zur Ódembildung Odembereitschaft des betreffenden Gewebes und Zufuhr von Elektrolyten, vorwiegend von $\mathrm{Na}$ exforderlich wären. Ferner hat $\mathrm{Fischer}{ }^{7)}$ auf Grund der Quellung der Kolloide durch Säuren die Säure-Ödemtheorie aufgestellt, wobei er annimmt, dass die Zunahme der H-Ionenkonzentration überhaupt Ödem verursache. Im Gegensatz hierzu behauptet Scha de ${ }^{8)}$, dass manche Ödeme durch Alkali bedingt wären. Hey ma $\mathrm{nn}^{91}$ konstatierte, dass im Durchspülungsversuch am Kaninchenohr Zusatz von Säuren oder Alkalien in die Spülfüssigkeit die Odembildung beschleunigt und $\mathrm{Kuwabata} \mathrm{a}^{10}$ ) bestätigte dabei, dass diese Neigung zur Ödembildung bei Alkalien stärker hervorgerufen werde. S a to ${ }^{11}$ hat nachgewiesen, dass Neigung zur Quellung des Kaninchenmuskels, der in 0,9\% iger $\mathrm{NaCl}$-Lösung eingetaucht worden ist, durch Verschiebung von $\mathrm{pH}$ des Mediums nach sowohl saurer als auch alkalischer Seite an Grad zunimmt. 
Bei durch Überventilation herbeigeführter Alkalosis fand Völ ke ${ }^{12)}$ Zunahme des Hämoglobingehaltes des Blutes, woraus er die Eindickung des Blutes schloss. Weiter haben Gollwitzer-Meier und $\mathrm{Chr}$. Mayer ${ }^{13}$ die durch Uberventilation hervorgerufenen Veränderungen der Blutkonzentration bei gesunden Menschen und Hunden vielfach untersucht, wobei sie fanden, dass bei Menschen Blutverdünnung. auftritt, wogegen bei Hunden sich das Blut eindickt. Kimura ${ }^{14}$ hat bei solchen Hunden Abnahme des Hämoglobingehaltes nachgewiesen. Vor kurzem haben Harkins und Hasting s ${ }^{15}$ ) bei Hunden Abnahme des Wassergehaltes im Serum nach intravenöser Injektion von Normal-HCl-Lösung in der Meñge von $31 / 3 \mathrm{ccm}$ pro kg Körpergewicht festgestellt; ferner beobachtete Laubender ${ }^{16)}$ an Kaninchen Bluteindickung nach peroraler Zufuhr von Salzsäurelösung. Ku ge $\mathrm{e}^{177}$ fand an Kaninchen bei Blutazidosis Abnahme des Hämoglobingehaltes und der Erythrozytenzahl, hingegen bei Blutalkalosis leichte Zunahme derselben. $O \mathrm{ka}^{18)}$ konstatierte bei Kaninchen bei weitem stärkere Abnahme der zirkulierenden Blut- und Plasmamenge sowie des Erythrozytenvolumens nach intravenöser Verabreichung von Säure- bzw. Alkalilösung in Mengen von $10 \mathrm{ccm}$ pro $\mathrm{kg}$ Körpergewicht als dieselben nach intravaskuläre Einverleibung der gleichen Menge von pysiologischer Kochsalzlösung.

Aus den obengenannten Resultaten geht schon hervor, dass die Veränderungen der $H$-Ionenkonzentration im Blute verschiedene Einflüsse auf die Konzentration sowie die chemisch-physikalischen Beschaffenheiten des Blutes ausüben müssen, aber es liegen recht spärlich die Untersuchungen vor, die sich mit dem Studium über den Einfluss der pH-Veränderung des Blutes auf das Bluteiweiss, insbesondere auf dessen Molekularaggregate befassen, und die Versuchsergebnisse sind zwischen den Autoren ziemlich divergierend.

Ozawa, Aikawa u. Arakawa ${ }^{19)}$ beobachteten bei Hunden relative Zunahme des Globulins im Serum im azidotischen Zustand nach intravenöser Einspritzung von Salzsäurelösung, Nisimu ra u. Ta kaga ki $\mathrm{i}^{20}$ haben bei Kaninchen Beschleunigung der Blutsenkungsgeschwindigkeit nach Salzsäure- oder Ammoniumchloridgabe berichtet.

Was die Veränderungen der Bluteiweisskörper bei der durch $\mathrm{O}_{2}$-Mangel herbeigeführten Acidosis anbelangt, sah $\mathrm{T} \mathrm{th}^{21}$ ' bei Einatmung verdünnter Luft bald eine Zunahme, bald eine Abnahme des Globulins; Elias u. Ta ubenha u s22) haben bei Kaninchen, die unter dem Unterdruck 2-3 Tage lang gehalten wurden, eine beträchtliche Albuminzunahme und eine geringe Globulinabnahme nachgewiesen. Loe w $\mathrm{y}^{23)}$ erwähnt in seiner Monographie, dass die Eiweisskörper des Blutes in Höhenklima mannigfache Veränderungen erfahren. Kasuga ${ }^{24)}$ konnte bei Hunden, indem er sie die 9-10\% $\mathrm{O}_{2}$-haltige Luft für eine Stunde einatmen liess, im Blute eine Abnahme des Albumins, eine relative und absolute Zunahme des Globulins sowie ein Absinken des kolloid-osmotischen Drucks (im folgenden abgekürzt : k.o.D.) nachweisen. Sekiguti25) hat an Kaninchen in der Unterdruckkammer bei verschiedenen Graden des Unterdrucks den k.o.D. des Blutes bestimmt und kam zum Ergebnis, dass er beim Unterdruck, der einer Höhelage von 3,000 m Höhe entspricht, absinkt, während er bei einem über $6,000 \mathrm{~m}$ Höhe entsprechenden Unterdruck um- 
gekehrt erhöht ist. Miura ${ }^{26)}$ machte bei Kaninchen die Beobachtung, dass beim progressiv fortschreitenden $\mathrm{O}_{2}$-Mangel der k.o.D. des Blutes absinkt, während er beim hochgradigen $\mathrm{O}_{2}$-Mangel hingegen eher erhöht ist. Is hi ka w a ${ }^{271}$ konstatierte, dass sich der k.o.D. sowie der k.p.D. für $1 \%$ Eiweiss (Druck pro \%) des Blutes beim langdauernden $\mathrm{O}_{2}$-Mangel erniedrigen.

Aus den Versuchsresultaten der obigen Autoren kann man Schluss ziehen, dass wenn man dem Organismus durch Säure- bzw. Alkalizufuhr einen azidotischen bzw. alkalotischen Zustand hervorbringt, im Zusammenhang mit Veränderungen des Wasseraustausches zwischen Blut und Gewebe quantitative sowie qualitative Veränderungen des Bluteiweisses, demgẹmäss auch Veränderungen des k.o.D. auftreten müssen. Darüber klar zu machen, dürfte irgend einen Beitrag zu den klinisch-therapeutischen Richtungen leisten. Nun, um mich darüber zu orientieren, ob und wieweit das Bluteiweiss und dessen k.o.D. dann, wenn intravenöse Injektion von Säuren oder Alkalien vorübergehende Veränderung der H-Ionenkonzentration im Blute hervorruft, verändert würde, habe ich in vorliegender Untersuchung zuerst bei gesunden Kaninchen, dänn bei Kaninchen mit Niereninsuffizienz die Bestimmungen ausgeführt und die Resultate miteinander verglichen.

Versuchsmethode: Als Versuchstier wurden gesunde Kaninchen von etwa $2 \mathrm{~kg}$ Körpergewicht, die 12 Stunden lang gehungert hatten, benutzt. In erster Versuchsreihe wurde dem Versuchstier Säure- bzw. Alkalilösung in Mengen von 10 ccm pro kg Körpergewicht intravenös injiziert, um Veränderungen der $\mathrm{H}$-Ionenkonzentration im Blute hervorzurufen. Hier bei kam als Säurelösung $0,9 \% \mathrm{NaCl}-$ haltige N/10-Salzsäurelösung und als Alkalilösung folgendes Gemisch zur Anwendung, welches nach dem Vorgang von $\mathrm{Gaza}$ und $\mathrm{B}$ randi ${ }^{28)}$ und $A$ uerbach u

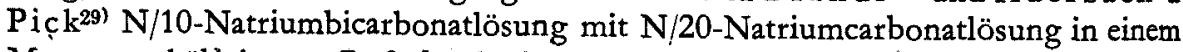
Mengenverhältinis von $7: 3$ durchmischt und dieses Gemisch mit einer $1,4 \%$ igen Kochsalzlösung im Verhältnis von 6:4 vermischt und zu isotonischer Alkalipufferlösung ( $\mathrm{pH}=9,30)$ hergestellt wurde. In der zweiten Versuchsreihe wurde derselbe Versuch bei Kaninchen mit Nierenfunktionsstörung ausgeführt. Um Funktionsstörung der Niere herbeizuführen, wurden die beiderseitigen Ureteren unterbunden, und das Tier wurde nach Ablauf von 50 Stunden nach dem Eingriff zum Versuch benutzt.

Jedes Versuchstier wurde im nüchternen Zustand auf einen elektrische passend angewärmten Tierhalter in der Rückenlage gefesselt und die A. femoralis auf der einen Seite blossgelegt, worin eine Kanüle eingelegen und sie behufs beliebiger Entnahme arteriellen Blutes mit einer Klemme gefasst worde. Um Einflüsse der Fesselung und Operation möglichst zu vermeiden, wurde die Blutentnahme erst nach zweistündigem Ruhiglassen der Versuchstiere vorgenommen. $30 \mathrm{~min}$ nach der Entnahme der ersten Blutprobe wurden die betreffenden körperwarmen Säure- oder Alkalilösungen in Mengn von $10 \mathrm{ccm}$ pro kg Körpergewicht mit einer Geschwindigkeit von etwa einer Minute in die Ohrvene injiziert und dann wurden die anderen Blutproben im direkten Anschluss daran (nach ungefähr $3 \mathrm{~min}$ ) und später nach 30 
sowie $60 \mathrm{~min}$, also insgesamt viermal, entnommen. In derselben Zeit jeder Blutentnahme bekam man kleine Blutmenge ohne Stauung aus der Ohrvene der anderen Seite unter Anwendung der von $\mathrm{Nak}$ a za wa und $\mathrm{Sat} \mathrm{o}^{11}$ ) ausgearbeiteten $\mathrm{M}$ is $\mathrm{low}$ i$\mathrm{tzer}^{30}$ schen Spritze, um das $\mathrm{pH}$ des Blutes mittels des Mis low itzer schen Potentiometers mit Chinhydronelektrode zu bestimmen. Des weiteren wurde an so gewonnenen Blutproben der Hämoglobingehalt mittelst des Fle is chel-M i e s ch e rschen Hämometers, der Serumeiweissgehalt mittelst des Pulfrichschen Eintauchrefraktometers, der k.o.D. nach der Methode von Krogh u. Nakazawa ${ }^{31}$ und der Fibrinogengehalt nach der Methode von Leendertz und Gromels ki ${ }^{32)}$ bestimmt.

Die entnommene gesamte Blutmenge, welche zu einmaliger Bestimmung erforderlich war, betrug ca. $2,5 \mathrm{ccm}$.

\section{Versuch an gesunden Kaninchen.}

1. Kontrolle. Injektion von physiologischer Kochsalzlösung.

Als Kontrolle für die Hauptversuche wurde 7 gesunden Kaninchen sterile körperwarme, 0,9\%ige Kochsalzlösung von gleicher Menge $(10 \mathrm{ccm}$ prokg Körpergewicht) wie Säure- bzw. Alkalilösung intravenös injiziert und die Schwankungen des Hämoglobin-; Serumeiweiss-, Fibrinogengehaltes und des k.o.D. sowie des $\mathrm{pH}$ Blutes wutden 30 min vor der Injektion, direkt nach derselben und später nach 30 sowie 60 min beobachtet. Die Versuchsresultate sind auf Tab 1. aufgetragen und nebénbei gibt Fig. 1 den durchschnittlichen prozentischen Wert der obigen Daten an.

Es wurde schon von Ito ${ }^{33)}$, Furuhasi, ${ }^{34)}$ Sasaki u. Yasuma ${ }^{35)}$ sowie Onoza ki ${ }^{36)}$ festgestellt, dass die Eiweiss- und Hämoglobinkonzentration des Blutes nach intravenöser Infusion von isotonischer Salzlösung eine vorübergehende Abnahme zeigt und dass diese Abnahme auf die Blutverdünnung zurückzuführen ist. Auch in meinen Versuchen kann man eine erhebliche Verminderung des Serumeiweiss- und Hämöglobingehaltes unmittelbar nach der Injektion von Kochsalzlösung ersehen; die prozentuale Abnahme des Hämoglobingehaltes betrug im Mittel 12,5. $(10,2-15,6) \%$ und die des Eiweissgehaltes im Mittel $12,3(9,8-14,5) \%$, also handelt es sich hierbei um eine nahezu parallele Konzentrationsverminderung der beiden Blutbestandteile. Nach dieser raschen Abnahme neigten die Konzentrationen des Eiweisses und des Hämoglobins in allen Fällen zur Erholung; nach 30 min näherten sich beide dem Vorwert, um dann aber nach $60 \mathrm{~min}$ wieder einigermassen abzusinken.

Betrachtet man nun die Schwankungen des K.o.D. des Blutes nach der Injektion, so kann man auf den ersten Blick leicht erkennen, dass sie mit denselben des Eiweiss- und Hämoglobingehaltes gleichen Schritt hielten; der k.o.D. nahm direkt nach der Infusion stürmisch $a b$, die prozentuelle Abnah- 
Tabelle 1.

Veränderungen des Bluteiweisses und dessen k.o.D. nach intravenöser Injektion von physiologischer Kochsalzlösung bei gesunden Kaninchen.

\begin{tabular}{|c|c|c|c|c|c|c|c|c|c|c|c|c|c|c|}
\hline & 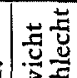 & & & & & $\begin{array}{l}\text { mei- } \\
\text { iss }\end{array}$ & & C.o.D. & & $\begin{array}{l}\text { ruck } \\
\text { ro } \%\end{array}$ & & $\begin{array}{l}\text { ibri- } \\
\text { logen }\end{array}$ & & $\begin{array}{l}\mathrm{H} \text { des } \\
\text { Blutes }\end{array}$ \\
\hline & 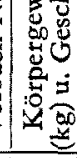 & $\begin{array}{c}\text { Zeit der } \\
\text { Blutentnahme }\end{array}$ & $\mathrm{g} / \mathrm{dl}$ & 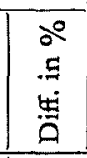 & $\%$ & 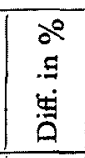 & 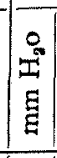 & 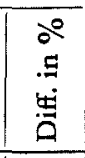 & 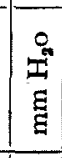 & $\mid \begin{array}{l}0 \\
. \pm \\
0 \\
0\end{array}$ & 20 & 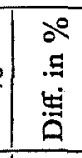 & & 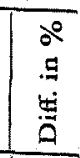 \\
\hline 1 & $\mid \begin{array}{c}2,150 \\
\text { 영 }\end{array}$ & $\begin{array}{l}\text { Vot } 30 \text { Min. } \\
\text { Direkt nach Infusion } \\
30 \text { Min. " } \\
60 \text { Min. " }\end{array}$ & $\begin{array}{l}13,36 \\
11,66 \\
12,80 \\
12,80\end{array}$ & $\mid \begin{array}{r}-12,7 \\
-4,2 \\
-4,2\end{array}$ & $\left|\begin{array}{l}5,40 \\
4,81 \\
5,07 \\
5,07\end{array}\right|$ & $\begin{array}{l}-10 \\
-6 \\
-6\end{array}$ & $\left|\begin{array}{l}239 \\
212 \\
224 \\
226\end{array}\right|$ & $\begin{array}{r}-11,3 \\
-6,3 \\
-5,4 \\
\end{array}$ & $\begin{array}{l}44,2 \\
44,1 \\
44,2 \\
44,5\end{array}$ & $\begin{array}{ll}- & 0,2 \\
\pm & 0 \\
+ & 0,7\end{array}$ & $\left\{\begin{array}{l}0,17 \\
0,11 \\
0,15 \\
0,15\end{array} \mid\right.$ & $\begin{array}{l}7 \\
1 \\
5 \\
5 \\
-11 \\
-11,\end{array}$ & $\begin{array}{r}7,30 \\
37,32\end{array}$ & $\begin{array}{l}+0,27 \\
\pm 0 \\
+0,14\end{array}$ \\
\hline 2 & $\mid \begin{array}{c}2,080 \\
\delta\end{array}$ & $\begin{array}{l}\text { Vor } 30 \mathrm{Min} . \\
\text { Direkt nach Infusion } \\
30 \mathrm{Min} . " \\
60 \mathrm{Min} . "\end{array}$ & $\left|\begin{array}{l}13,08 \\
11,66 \\
12,24 \\
12,20\end{array}\right|$ & $\left|\begin{array}{r}-10,8 \\
-6,4 \\
-6,7\end{array}\right|$ & $\mid \begin{array}{l}6,19 \\
5,58 \\
5,79 \\
5,66\end{array}$ & $\left|\begin{array}{r}-9,8 \\
-7,3 \\
-9,6\end{array}\right|$ & $\left|\begin{array}{l}259 \\
232 \\
240 \\
234\end{array}\right|$ & $\left|\begin{array}{r} \\
-10,4 \\
-7,3 \\
-9,6\end{array}\right|$ & $\left|\begin{array}{l}41,8 \\
41,6 \\
41,4 \\
41,3\end{array}\right|$ & $\mid \begin{array}{r} \\
-0,5 \\
-0,9 \\
-1,4\end{array}$ & 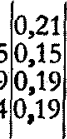 & $\mid \begin{array}{r}-28,6 \\
-9,5 \\
-9,5\end{array}$ & $\begin{array}{l}7,36 \\
7,34 \\
7,36 \\
7,36\end{array}$ & $\begin{array}{l}-0,27 \\
\pm 0 \\
\pm 0\end{array}$ \\
\hline 3 & 1,800 & $\begin{array}{l}\text { Vor } 30 \text { Min. } \\
\text { Direkt nach Infusion } \\
30 \text { Min. " } \\
60 \mathrm{Min} . "\end{array}$ & $\begin{array}{l}13,94 \\
12,30 \\
13,08 \\
12,80\end{array}$ & $\begin{array}{r}-11,8 \\
-6,2 \\
-8,2 \\
\end{array}$ & $\begin{array}{l}6,40 \\
5,60 \\
5,92 \\
5,76\end{array}$ & $\begin{array}{r}-12,5 \\
-7,5 \\
-10,0\end{array}$ & \begin{tabular}{|l|}
266 \\
232 \\
246 \\
245
\end{tabular} & $\begin{array}{r}-14,3 \\
-7,5 \\
-7,9 \\
\end{array}$ & $\begin{array}{l}41,6 \\
41,4 \\
41,5 \\
42,5\end{array}$ & $\begin{array}{r}-0 \\
-0 \\
+2\end{array}$ & $\left\{\begin{array}{l}0,17 \\
0,13 \\
0,15 \\
0,15\end{array}\right.$ & $\begin{array}{l}-23,5 \\
-11,8 \\
-11,8\end{array}$ & $\left|\begin{array}{l}7,39 \\
7,39 \\
7,39 \\
7,37\end{array}\right|$ & $\begin{array}{l}+0,27 \\
+0,27 \\
\pm 0\end{array}$ \\
\hline 4 & $\left|\begin{array}{c}1,500 \\
7\end{array}\right|$ & $\begin{array}{l}\text { Vor } 30 \text { Min. } \\
\text { Direkt nach Infusion } \\
30 \text { Min. " } \\
60 \text { Min. " }\end{array}$ & $\left|\begin{array}{l}12,80 \\
10,80 \\
11,96 \\
11,96\end{array}\right|$ & $\begin{array}{r} \\
-15,6 \\
-6,5 \\
-6,5 \\
\end{array}$ & $\mid \begin{array}{l}6,40 \\
5,47 \\
5,75 \\
5,70 \\
1\end{array}$ & $\mid \begin{array}{r}\mid \\
-14,5 \\
-10,1 \\
-10,9\end{array}$ & $\begin{array}{l}249 \\
210 \\
225 \\
222 \\
\end{array}$ & $\begin{array}{r}-15,7 \\
-9,6 \\
-10,8\end{array}$ & $\begin{array}{l}38,9 \\
38,4 \\
39,1 \\
38,9\end{array}$ & $\begin{array}{l}-1,3 \\
+0 \\
\pm 0\end{array}$ & $\begin{array}{l}0,15 \\
0,11 \\
0,13 \\
0,13\end{array}$ & $\begin{array}{r}-23,3 \\
-13,3 \\
-13,3\end{array}$ & 7,37 & $\begin{array}{l}+0,14 \\
\pm 0 \\
\pm 0\end{array}$ \\
\hline 5 & 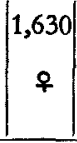 & $\begin{array}{l}\text { Vor } 30 \text { Min. } \\
\text { Direkt nach Infusion } \\
30 \mathrm{Min} . " \\
60 \mathrm{Min} . "\end{array}$ & $\begin{array}{l}13,36 \\
11,36 \\
12,80 \\
12,52\end{array} \mid$ & $\begin{array}{r} \\
-15,0 \\
-4,2 \\
-6,3 \\
\end{array}$ & $\begin{array}{l}7,18 \\
6,14 \\
6,62 \\
6,57\end{array}$ & $\begin{array}{r}\mid 14,5 \\
-7,8 \\
-8,5 \\
\end{array}$ & \begin{tabular}{|l|}
297 \\
252 \\
274 \\
274
\end{tabular} & $\begin{array}{r}\mid \\
-15,1 \\
-7,7 \\
-7,7\end{array}$ & $\mid \begin{array}{l}41,4 \\
41,0 \\
41,4 \\
41,3\end{array}$ & $\left|\begin{array}{rr}- & \\
- & 1,0 \\
+ & 0 \\
- & 0,2\end{array}\right|$ & $\begin{array}{l}0,21 \\
0,15 \\
0,19 \\
0,19\end{array}$ & $\begin{array}{r} \\
-28,6 \\
-9,5 \\
-9,5\end{array}$ & $\mid \begin{array}{l}7,31 \\
7,31 \\
7,30 \\
7,31\end{array}$ & $\begin{array}{l}+0,14 \\
\pm 0 \\
\pm 0\end{array}$ \\
\hline & $\left|\begin{array}{c}1,620 \\
\delta\end{array}\right|$ & $\begin{array}{l}\text { Vor } 30 \text { Min. } \\
\text { Direkt nach Infusion } \\
30 \text { Min. " } \\
60 \text { Min. } "\end{array}$ & $\left|\begin{array}{l}13,94 \\
12,52 \\
13,08 \\
12,80\end{array}\right|$ & $\mid \begin{array}{r}-10,2 \\
-6,2 \\
-8,2\end{array}$ & $\begin{array}{l}6,64 \\
5,90 \\
6,14 \\
6,12\end{array}$ & $\begin{array}{r}-11,1 \\
-7,5 \\
\end{array}$ & $\left|\begin{array}{l}284 \\
250 \\
260 \\
258\end{array}\right|$ & $\begin{array}{r} \\
-12,0 \\
-8,4 \\
-9,1\end{array}$ & $\begin{array}{l}42,3 \\
42,4 \\
42,3 \\
42,1\end{array}$ & 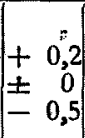 & $\mid \begin{array}{l}0,24 \\
0,19 \\
0,21 \\
0,21\end{array}$ & $\begin{array}{l}-20 \\
-12 \\
-12\end{array}$ & $\begin{array}{r}33 \\
31 \\
332 \\
33\end{array}$ & $\begin{array}{l}-0,27 \\
-0,14 \\
\pm 0\end{array}$ \\
\hline & $\left|\begin{array}{c}1,650 \\
7\end{array}\right|$ & $\begin{array}{l}\text { Vor } 30 \text { Min. } \\
\text { Ditekt nach Infusion } \\
30 \text { Min. " } \\
60 \text { Min. " }\end{array}$ & $\mid \begin{array}{l}14,24 \\
12,52 \\
13,64 \\
13,36\end{array}$ & $\begin{array}{r}-12,1 \\
-4,2 \\
-6,2\end{array}$ & $\begin{array}{l}6,27 \\
5,49 \\
5,94 \\
5,72\end{array}$ & $\begin{array}{r} \\
-12,4 \\
-5,3 \\
-8,8\end{array}$ & \begin{tabular}{|l}
264 \\
230 \\
246 \\
245
\end{tabular} & $\begin{array}{r}-12,9 \\
-6,8 \\
-7,2\end{array}$ & $\begin{array}{l}42,1 \\
41,9 \\
42,1 \\
42,8\end{array}$ & 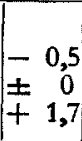 & $\mid \begin{array}{l}0,21 \\
0,15 \\
0,19 \\
0,17\end{array}$ & $\mid \begin{array}{r}-28 \\
-14 \\
-19\end{array}$ & $\mid \begin{array}{l}7,28 \\
7,29 \\
7,28 \\
7,28\end{array}$ & $\begin{array}{l}+0,14 \\
\pm 0 \\
\pm 0\end{array}$ \\
\hline & Е & $\begin{array}{l}\text { Vor } 30 \text { Min. } \\
\text { Direkt nach Infusion } \\
30 \text { Min. } \\
60 \text { Min. }\end{array}$ & $\begin{array}{l}13,53 \\
11,83 \\
12,80 \\
12,63\end{array}$ & $\begin{array}{r}-12 \\
-5 \\
-6\end{array}$ & $\begin{array}{l}6,35 \\
5,57 \\
5,89\end{array}$ & 12 & $\left|\begin{array}{l}265 \\
231 \\
246\end{array}\right|$ & $\begin{array}{r} \\
-12 \\
-7\end{array}$ & $\begin{array}{l}41,8 \\
41,5 \\
41,7\end{array}$ & $\begin{array}{r}-0 \\
-0 \\
+0\end{array}$ & $\begin{array}{l}0,19 \\
0,14 \\
0,17\end{array}$ & $\begin{array}{r}-2 \\
-1 \\
+1\end{array}$ & $\begin{array}{l}33 \\
, 32\end{array}$ & $\begin{array}{l}-0 \\
\pm\end{array}$ \\
\hline
\end{tabular}

me betrug durchschnittlich $12,8(10,4-15,7) \%$. Dann neigte ex etwas zur Erholung, aber nach Ablauf von 60 min zeigte er wieder mehr oder weniger eine Abnahme. Wie aus Fig. 1 ersichtlich, gehen das Serumeiweiss und der k.o.D. stets miteinander Hand in Hand, demgemäss der Druck pro \% nur bescheidenen Schwankungen unterworfen ist. 
Fig. 1. Prozentuelle Blutveränderungen nach intravenöser Injektion von physiologischer Kochsalzlösung bei gesunden Kaninchen (Durchschnitt).

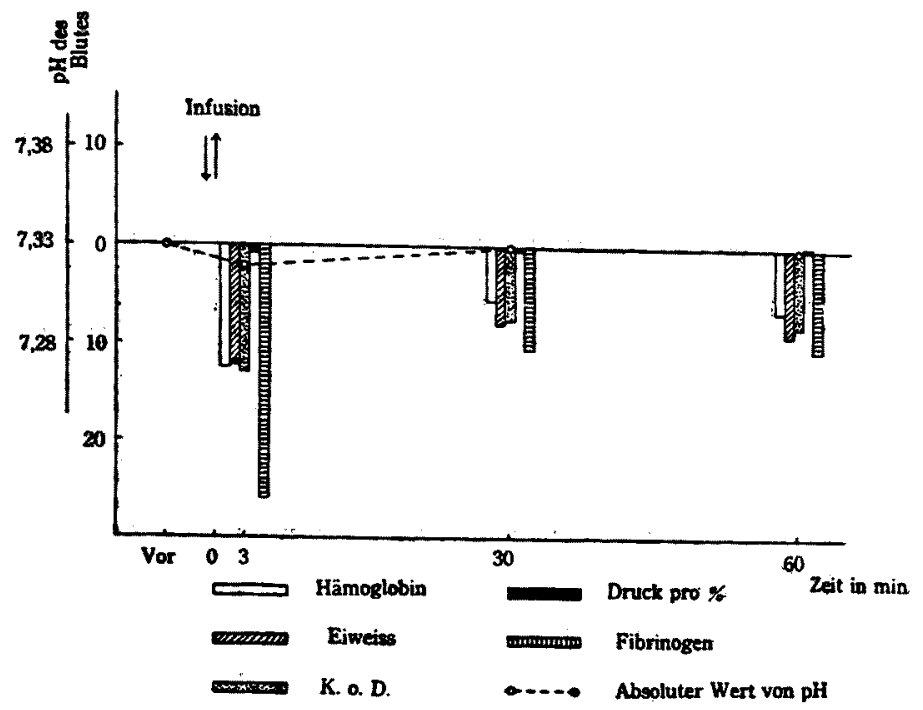

Der Fibrinogengehalt nahm auch direkt nach der Injektion exstaunlich ab, die Abnahme bettug im Durchschnitt 26,3 (20,8-35,3)\%, dann zeigte er aber deutliche Wiederherstellung und hielt mit dem Eiweiss fast gleichen Schritt. Das pH des Blutes erfuhr nach der Injektion keine bemerkbare Schwankung:

Die im direkten Anschluss an die Injektion erscheinende erhebliche Abnahme des Eiweisses, des Hämoglobins, des k.o.D. und des Fibrinogens ist selbstyerständlich der durch infundierte Kochsalzlösung hervorgerufenen Blutverdünnung und die darauffolgende Wiederzunahme derselben der auf dem extravasalen Austritt der infundierten Flüssigkeit beruhenden Bluteindickung zuzuschreiben, während die nach 60 min eintretende geringe Verminderung derselben offenbar auf die wiederholentlich ausgefijhrte Blutentnahme zurückzuführen ist.

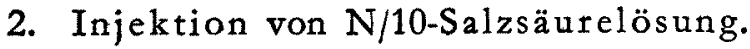

Es wurde $\mathrm{N} / 10$-Salzsäurelösung, in deren Deziliter $0,9 \mathrm{~g} \mathrm{NaCl}$ aufgelöst wurde, auf die Körpertemperatur erwärmt, gesunden Kaninchen in Mengen von $10 \mathrm{ccm}$ pro $\mathrm{kg}$ Körpergewicht intravenös einverleibt. Danach wurden die Blutproben in gleichen Zeitabständen wie im Kontrollversuch entnommen und zu Bestimmungen benutzt. Die Ergebnisse der 9 Versuche sind in Tab. 2 wiedergegeben; Fig. 2 zeigt die prozentualen Ab- und Zunahme der Bestimmungswerten im Durchschnitt. 
Tabelle 2.

Veränderungen des Bluteiweisses und dessen k.o.D. nach intravenöser Injektion von $\mathrm{N} / 10$ Salzsäurelösung bei gesunden Kaninchen.

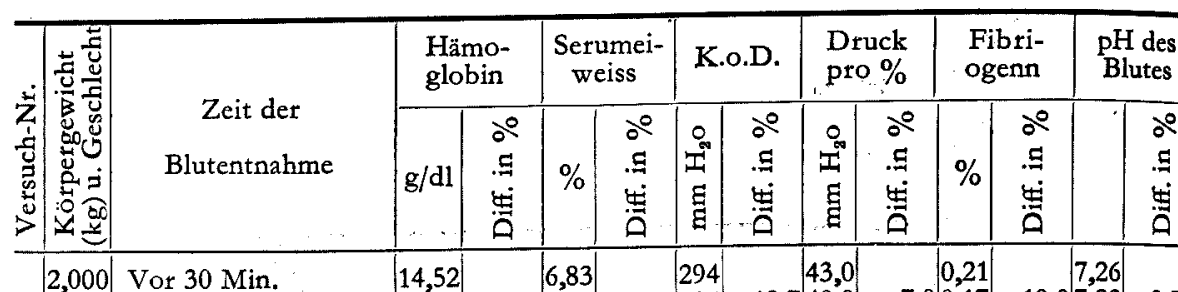

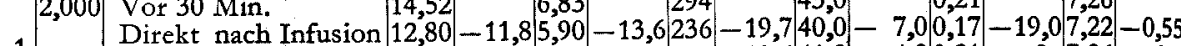

우 30 Min. $n \quad 13,64-6,16,16-9,8 \mid 254-13,641,2-4,2[0,21 \pm 0 \quad 7,26 \pm 0$ $60 \mathrm{Min.} " \quad \Rightarrow \quad 13,36-8,06,12-10,4|256-12,9| 41,8-2,8|0,21| \pm 0 \quad 7,27 \mid+0,14$

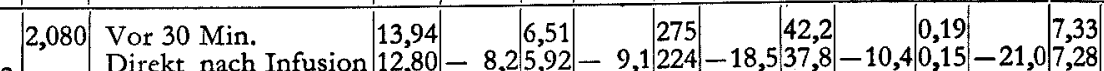

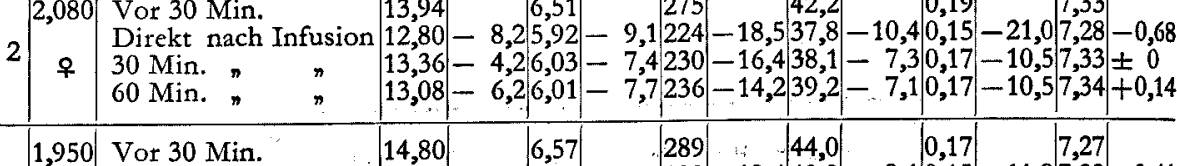

3 Direkt nach Infusion $|13,36|-9,75,83|-11,3| 233|-19,4| 40,0-9,1|0,15|-11,8|7,23|-0,41$

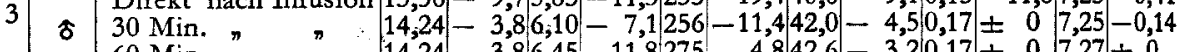
$60 \mathrm{Min.} \quad " \quad " \quad|14,24|-3,86,45-11,8|275|-4,8|42,6|-3,2|0,17| \pm 0|7,27| \pm 0$

1 1,750| Vor $30 \mathrm{Min}, \quad-|12,80| \quad|6,49| \quad|287| \quad|44,2| \quad|0,15| \quad|7,32|$

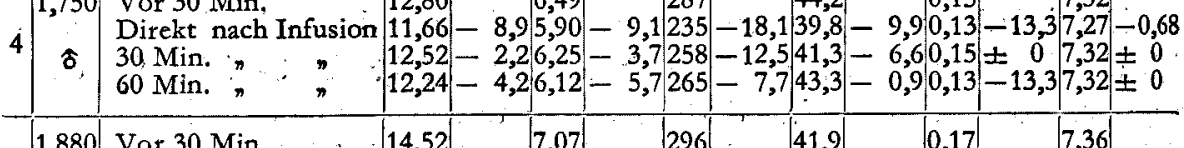

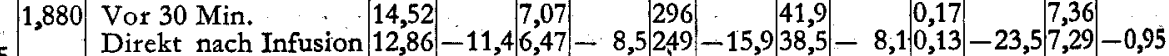

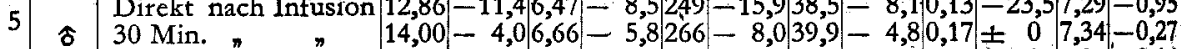
60 Min. " ". $14,00-4,06,80-3,8280-5,441,2-1,70,17 \pm 07,37+0,14$

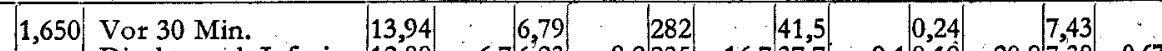

6 Direkt nach Infusion $12,80-6,7|6,23-8,2| 235-16,7 \mid 37,7-9,10,19-20,87,38-0,67$

우 30 Min. $, \quad 13,36-4,26,47-4,7263-6,740,5-2,40,24 \pm 07,41-0,27$

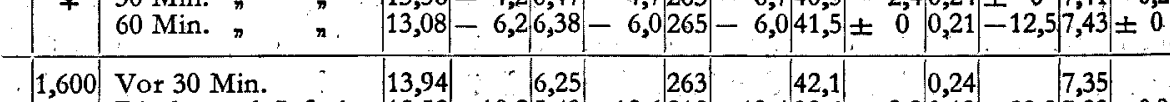

7 Direkt nach Infusion $12,52-10,25,49-12,6 \mid 212-19,438,6-8,30,19-20,87,28-0,95$

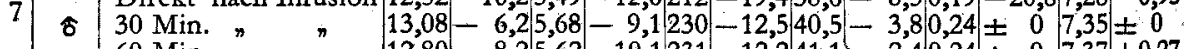

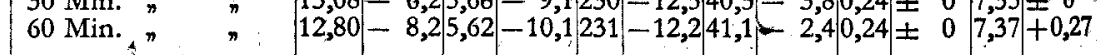

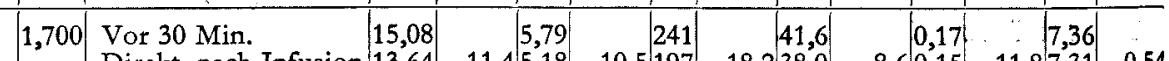

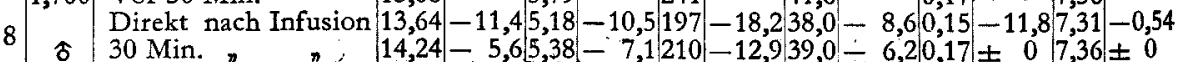

$\pi \quad 14,24-5,65,38-7,1210-12,939,0 \div 6,20,17 \pm 07,36 \pm 0$ 60 Min. " " $\quad 13,94 \mid-7,5[5,22|-9,8| 210|-12,9| 41,4-4,8|0,17| \pm 0|7,36| \pm 0$

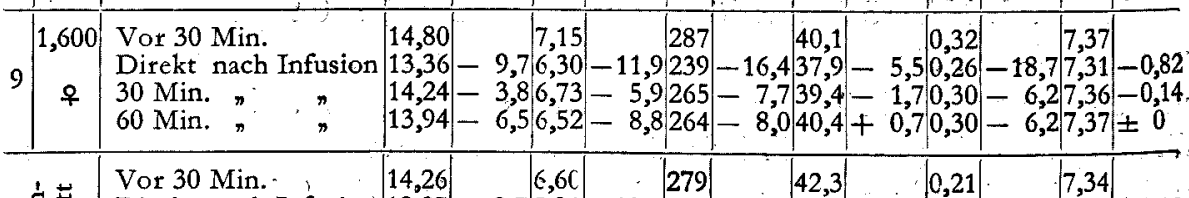

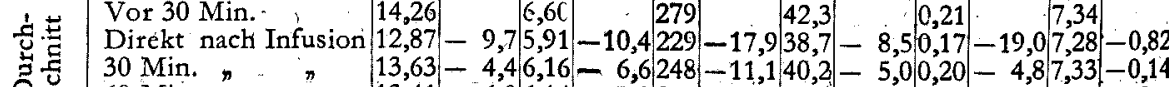

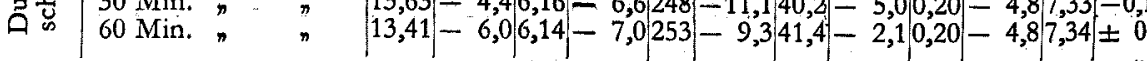


Das $\mathrm{pH}$ des Blutes nahm direkt nach der Injektion ein wenig ab, aber nach 30 min kehrte es im grossen und ganzen zum Anfangswert zurück; der Einfluss der Säurezufuhr war nämlich vorübergehend.

Fig. 2. Prozentuelle Blutveränderungen nach intravenöser Injektion von $\mathrm{N} /{ }_{10}$-HCl-Lösung bei gesunden Kaninchen (Durchsehnitt).

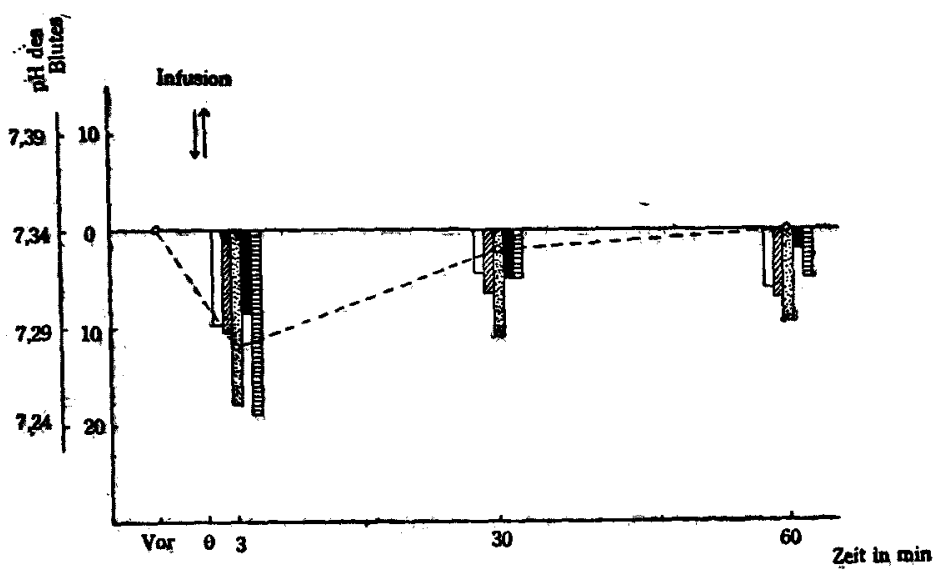

Zeichenerklärungen wie bei Fig. 1.

Unmittelbar nach der Injektion betrug die durchschnittliche Abnahme beim Hämoglobingehalt $9,7(6,7-11,8) \%$, beim Eiweissgehalt $10,4(8,2-$ 15,4) \%, beim Fibrinogengehalt 19,0 (11,8-23,5) \% und beim k.o.D. 17,9 $(15,9-19,7) \%$. Wie aus diesen Zifferzahlen ersichtlich, war die Abnahme des Hämoglobin-, Eiweiss- und Fibrinogengehaltes kleiner und die des k.o.D. hingegen bettächtlich grösser als die im Kontrollversuch, folglich nahm der Druck pro \% augenfällig ab; die Abnahme desselben betrug im Mittel 8,5 $(7,0-10,4) \%$. Nach 30 min näherten sich der Eiweiss- und Fibrinogengehalt mit der Konzentrationssteigerung des Hämoglobins zum Anfangswert und nach 60 min nahmen sie wịeder ein wenig ab. Im Gegensatz dazu blieb der k,o.D. erniedrigt ; im Mittel betrug seine Abnahme nach 30 min 11,1 (6,7$16,4) \%$, nach 60 min noch $9,3(4,8-14,2) \%$, infolgedessen belief sich die durchschnittliche Abnahme des Drucks pro \% nach $30 \mathrm{~min}$ auf $5,0(1,7-7,3)$ $\%$ und nach $60 \mathrm{~min}$ auf $2,1(0,7-7,1) \%$.

3. Injektion von isotonischer Alkalipufferlösung.

Bei 8 gesuriden Kaninchen wurde die intravenöse Injektion von isotonischer Alkalipufferlösung $\left(\mathrm{pH}_{F}=9,30\right)$ in Mengen von $10 \mathrm{ccm}$ pro kg Körpergewicht durchgeführt. Die Versuchsergebnisse sind in Tab. 3 zusammengestellt, Fig. 3 zeigt die durchschnittlichen prozentualen Werte derselben.

Das $\mathrm{pH}$ des Blutes nahm direkt nach der Injektion vorübergehend mässig 
zu, dann fing es an, allmählich abzusteigen, um nach 60 Min. fast zum Vorwert zurückzukehren.

\section{Tabelle 3.}

Veränderungen des Bluteiweisses und dessen k.o.D. nach intravenöser Injektion von isotonischer Alkalipufferlösung $(\mathrm{pH}=9,30)$ bei gesunden Kaninchen.

\begin{tabular}{|c|c|c|c|c|c|c|c|c|c|c|c|c|c|c|}
\hline & 点荳 & & & & & & & D. & & & & & & \\
\hline & 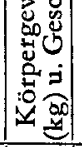 & $\begin{array}{l}\text { Zeit der } \\
\text { Blutentnahme }\end{array}$ & $\mathrm{g} / \mathrm{dl}$ & $\begin{array}{l}00 \\
\dot{a} \\
\dot{A} \\
\dot{a}\end{array}$ & $\%$ & 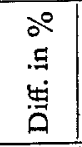 & 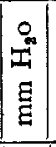 & 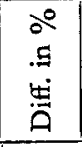 & $\begin{array}{c}0 \\
\stackrel{్}{I} \\
E \\
E \\
E\end{array}$ & 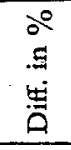 & $\%$ & $\begin{array}{l}20 \\
.5 \\
0 \\
0\end{array}$ & & 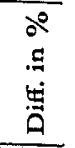 \\
\hline 1 & $\begin{array}{c}1,900 \\
8\end{array}$ & $\begin{array}{l}\text { Vor } 30 \mathrm{Min} . \\
\text { Direkt nach Infusion } \\
30 \mathrm{Min} . " \\
60 \mathrm{Min} . "\end{array}$ & $\begin{array}{l}14,52 \\
12,80 \\
13,64 \\
13,64\end{array} \mid$ & $\begin{array}{l}-11 \\
-6 \\
-6\end{array}$ & $\left|\begin{array}{l}7,18 \\
6,34 \\
6,66 \\
6,68\end{array}\right|$ & $\begin{array}{r}-11,7 \\
-7,2 \\
-7,0\end{array}$ & $\begin{array}{l}290 \\
241 \\
255 \\
258\end{array}$ & $\begin{array}{r}-16,9 \\
-12,1 \\
-11,0\end{array}$ & $\begin{array}{l}40,4 \\
38,0 \\
38,3 \\
38,6\end{array}$ & $\begin{array}{r} \\
-5 \\
-5 \\
-4 \\
\end{array}$ & $\begin{array}{l}0,21 \\
0,17 \\
0,21 \\
0,19\end{array}$ & $\mid \begin{array}{cc} & \\
-19,1 \\
\pm & 0 \\
- & 9,5\end{array}$ & $\begin{array}{l}7,39 \\
7,37 \\
7,34\end{array}$ & $\begin{array}{l}+ \\
+0 \\
+0 \\
+0\end{array}$ \\
\hline 2 & $\left|\begin{array}{c}1,820 \\
8\end{array}\right|$ & $\begin{array}{l}\text { Vor } 30 \text { Min. } \\
\text { Direkt nach Infusion } \\
30 \mathrm{Min} . " n \\
60 \mathrm{Min} . " n\end{array}$ & $\begin{array}{l}13,64 \\
12,52 \\
13,36 \\
13,08\end{array}$ & $\begin{array}{r}-8 \\
-2 \\
-4\end{array}$ & $\begin{array}{l}7,24 \\
6,41 \\
6,90 \\
6,86\end{array}$ & $\begin{array}{r} \\
-11,5 \\
-4,7 \\
-5,2 \\
\end{array}$ & $\left|\begin{array}{l}315 \\
263 \\
294 \\
293\end{array}\right|$ & $\begin{array}{r} \\
-16,5 \\
-6,7 \\
-7,0\end{array}$ & $\mid \begin{array}{l}43,5 \\
41,0 \\
42,6 \\
42,7\end{array}$ & $\begin{array}{r} \\
-5 \\
-2 \\
-1 \\
\end{array}$ & $\begin{array}{l}0,19 \\
70,15 \\
10,17 \\
0,17 \\
0,\end{array}$ & $\begin{array}{r}-21,0 \\
-10,5 \\
-10,5 \\
\end{array}$ & $=$ & $\begin{array}{l}+0,95 \\
+0,41 \\
\pm 0\end{array}$ \\
\hline 3 & 1,890 & $\begin{array}{l}\text { Vor } 30 \text { Min. } \\
\text { Direkt nach Infusion } \\
30 \text { Min. } \\
60 \text { Min. }\end{array}$ & $\begin{array}{l}13,94 \\
12,24 \\
13,08 \\
13,08\end{array} \mid$ & $\begin{array}{r} \\
-12,2 \\
-6,2 \\
-6,2\end{array}$ & $\begin{array}{l}7,46 \\
6,34 \\
6,95 \\
6,77\end{array}$ & $\begin{array}{r} \\
-15,0 \\
-6,8 \\
-9,2\end{array}$ & $\left|\begin{array}{l}309 \\
255 \\
280 \\
274\end{array}\right|$ & $\begin{array}{r}-17,5 \\
-9,4 \\
-11,3\end{array}$ & $\begin{array}{l}41,4 \\
40,2 \\
40,3 \\
40,5\end{array}$ & $\begin{array}{r}-2 \\
-2 \\
-2\end{array}$ & $\mid \begin{array}{l}0,26 \\
0,21 \\
0,24 \\
0,24\end{array}$ & $\left|\begin{array}{r} \\
-23,5 \\
-7,7 \\
-7,7\end{array}\right|$ & $\mid \begin{array}{l}7,36 \\
7,41 \\
7,38\end{array}$ & $\begin{array}{r} \\
+0,68 \\
+0,27 \\
+0,13\end{array}$ \\
\hline 4 & $\begin{array}{l}800 \\
\text { 今 }\end{array}$ & $\begin{array}{l}\text { Vor } 30 \text { Min. } \\
\text { Direkt nach Infusion } \\
30 \mathrm{Min} . "{ }^{n} \\
60 \mathrm{Min} . "\end{array}$ & $\begin{array}{l}14,24 \\
12,80 \\
13,64 \\
13,36\end{array}$ & $\begin{array}{l}- \\
-10, \\
-4,2 \\
-6,2\end{array}$ & $\left\{\begin{array}{l}7,07 \\
6,12 \\
6,66 \\
6,62\end{array}\right.$ & $\begin{array}{r} \\
-13,4 \\
-5,8 \\
-6,4\end{array}$ & $\mid \begin{array}{l}282 \\
230 \\
258 \\
262\end{array}$ & $\begin{array}{r}-18,4 \\
-8,5 \\
-7,1\end{array}$ & $\begin{array}{l}39,9 \\
37,6 \\
38,7 \\
39,6\end{array}$ & $\begin{array}{r} \\
-5,8 \\
-3,0 \\
-0,7\end{array}$ & $\begin{array}{l}0,15 \\
0,13 \\
0,15 \\
0,13\end{array}$ & $\begin{array}{c}-13,3 \\
\pm 0 \\
-13,3\end{array}$ & \begin{tabular}{|l|}
7,43 \\
7,50
\end{tabular} & $+0,94$ \\
\hline 5 & 1,870 & $\begin{array}{l}\text { Vor } 30 \text { Min. } \\
\text { Direkt nach Infusion } \\
30 \mathrm{Min} . " \\
60 \mathrm{Min} . "\end{array}$ & $\left|\begin{array}{l}14,80 \\
13,36 \\
14,24 \\
14,24\end{array}\right|$ & $\begin{array}{r}-9 \\
-3 \\
-3\end{array}$ & $\begin{array}{l}7,05 \\
6,34 \\
6,57 \\
6,62\end{array}$ & $\begin{array}{r}10,1 \\
-10,1 \\
-6,8 \\
-6,1\end{array}$ & $\begin{array}{l}278 \\
240 \\
253 \\
258\end{array}$ & $\begin{array}{r}-13,7 \\
-9,0 \\
-7,2\end{array}$ & $\begin{array}{l}39,4 \\
37,8 \\
38,5 \\
39,0\end{array}$ & $\begin{array}{r} \\
-4,1 \\
-2,3 \\
-\quad 1,0\end{array}$ & $\mid \begin{array}{l}0,24 \\
0,19 \\
0,22 \\
0,22 \\
0,2\end{array}$ & $\begin{array}{r}-20 \\
-8 \\
-8 \\
\end{array}$ & 7,39 & $\begin{array}{l}+0 \\
+0 \\
+0\end{array}$ \\
\hline 6 & 1,880 & $\begin{array}{l}\text { Vor } 30 \text { Min. } \\
\text { Direkt nach Infusion } \\
30 \text { Min. " } \\
60 \text { Min. " }\end{array}$ & $\begin{array}{l}14,24 \\
12,80 \\
13,64 \\
13,64\end{array}$ & $\begin{array}{l}-10 \\
-4 \\
-4 \\
\end{array}$ & $\mid \begin{array}{l}7,13 \\
6,38 \\
6,77 \\
6,73\end{array}$ & $\begin{array}{r}-10,5 \\
-5,0 \\
-5,6 \\
\end{array}$ & $\begin{array}{l}270 \\
231 \\
249 \\
250\end{array}$ & $\begin{array}{r}-14 \\
-7 \\
-7 \\
\end{array}$ & $\begin{array}{l}37,9 \\
36,2 \\
36,8 \\
37,1\end{array}$ & $\begin{array}{l}-4,5 \\
-2,5 \\
-2,1\end{array}$ & $\begin{array}{l}0,19 \\
0,15 \\
0,19 \\
0,19 \\
0,17\end{array}$ & $\begin{array}{l}-21,0 \\
\pm 0 \\
-10,5\end{array}$ & {$\left[\begin{array}{l}7,20 \\
7,37 \\
7,30 \\
7,28\end{array}\right.$} & $\begin{array}{l}+1,24 \\
+0,27 \\
\pm 0\end{array}$ \\
\hline 7 & 950 & $\begin{array}{l}\text { Vor } 30 \text { Min. } \\
\text { Direkt nach Infusion } \\
30 \text { Min. " } \\
60 \text { Min. }\end{array}$ & $\begin{array}{l}14,24 \\
12,80 \\
13,64 \\
13,36\end{array}$ & $\begin{array}{r} \\
-8, \\
-4, \\
-6,2\end{array}$ & $\left\{\begin{array}{l}6,68 \\
5,83 \\
6,25 \\
6,20\end{array}\right.$ & $\begin{array}{r} \\
-12,7 \\
-6,4 \\
-7,2 \\
\end{array}$ & $\begin{array}{l}264 \\
221 \\
242 \\
240\end{array}$ & $\begin{array}{r}-16,3 \\
-8,3 \\
-9,1 \\
\end{array}$ & $\begin{array}{l}39,5 \\
37,9 \\
38,7\end{array}$ & $\begin{array}{l}-4, \\
-4, \\
-2, \\
\end{array}$ & $\begin{array}{l}0,17 \\
0,13 \\
0,13 \\
0,17 \\
0,15\end{array}$ & $\left|\begin{array}{c}-23,5 \\
\pm 0 \\
-11,8\end{array}\right|$ & $\begin{array}{l}1,40 \\
7,49 \\
7,46\end{array}$ & $\begin{array}{l}+1 \\
+0 \\
+0\end{array}$ \\
\hline .8 & 10 & $\begin{array}{l}\text { Vor } 30 \text { Min. } \\
\text { Direkt nach Infusion } \\
30 \text { Min. } " \\
60 \text { Min. } "\end{array}$ & $\begin{array}{l}13,64 \\
12,24 \\
13,08 \\
13,08\end{array}$ & $\begin{array}{r}-10 \\
-4, \\
-4, \\
\end{array}$ & $\left|\begin{array}{l}6,77 \\
6,09 \\
6,45 \\
6,47\end{array}\right|$ & $\begin{array}{r}-10,0 \\
-4,7 \\
-4,4 \\
\end{array}$ & $\begin{array}{l}286 \\
242 \\
258 \\
268\end{array}$ & $\begin{array}{r}-12,9 \\
-9,8 \\
-6,3\end{array}$ & $\mid \begin{array}{l}42,2 \\
39,7 \\
40,0\end{array}$ & $\begin{array}{r}-5 \\
-5 \\
-5 \\
-1\end{array}$ & $\mid \begin{array}{l}0,17 \\
0,15 \\
0,17 \\
0,17 \\
0,17\end{array}$ & $\left|\begin{array}{ll} & \\
-11,8 \\
\pm & 0 \\
\pm & 0\end{array}\right|$ & $\mid \begin{array}{l}7,31 \\
7,37 \\
7,34 \\
7,31\end{array}$ & $\begin{array}{l}+0,82 \\
+0,27 \\
\pm 0\end{array}$ \\
\hline & 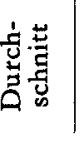 & $\begin{array}{l}\text { Vor } 30 \text { Min. } \\
\text { Direkt nach Infusion } \\
30 \text { Min. } \\
60 \text { Min. }\end{array}$ & $\left|\begin{array}{l}12,73 \\
13,54 \\
13,43\end{array}\right|$ & -1 & $\begin{array}{l}7,07 \\
6,23 \\
6,65\end{array}$ & $\begin{array}{l}-11 \\
-5 \\
-6\end{array}$ & 240 & -1 & 30 & 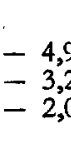 & $\left|\begin{array}{l}0,20 \\
0,16 \\
0,19\end{array}\right|$ & ? & $F$ & $i$ \\
\hline
\end{tabular}


Fig. 3. Prozentuelle Blutveränderungen nach intravenöser Injelttion von isotonischer Alkalipufferlösung ( $\mathrm{pH}=9,30)$ bei gesunden Kaninchen (Durchschnitt).

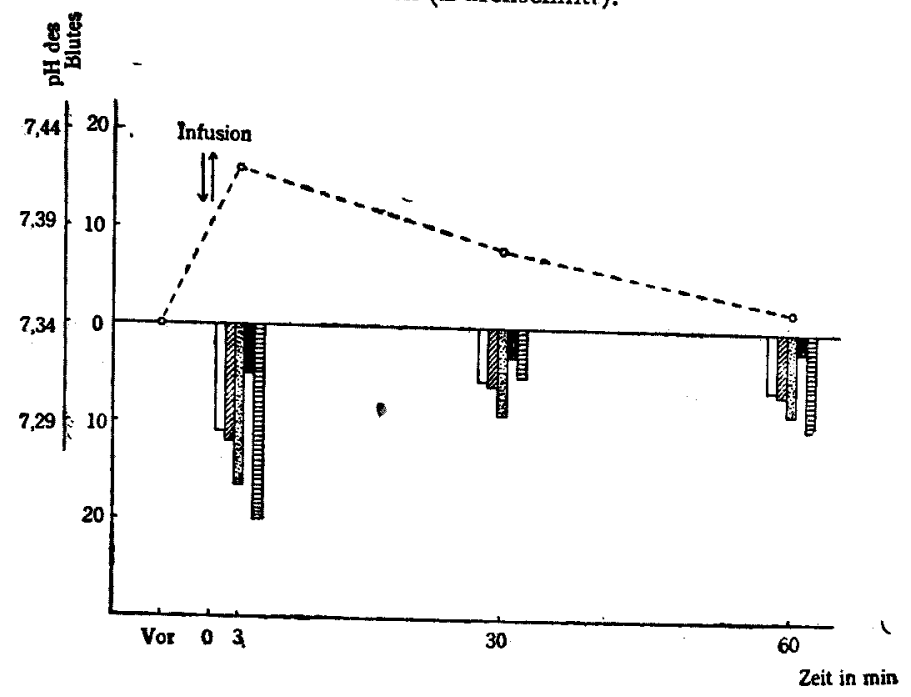

Zeichenerklärungen wie bei Fig. 1.

Unmittelbar nach der Injektion nahmen der Hämoglobin-, Eiweiss-und Fibrinogengehalt sowie der k.o.D. ziemlich ab; im Mittel betrug die Abnahme beim Hämoglobingehalt 10,8 $(8,1-12,2) \%$, beim Eiweissgehalt $11,9(10,0-$ $15,9) \%$, und beim Fibrinogengehalt $20,0(11,8-23,5) \%$; also ergaben sich diese Zahlen kleiner als die im Kontrollversuch. Hingegen erwies sich die Abnahme des k.o.D. als grösser als die im Kontrollversuch, demgemäss erfolgte die Abnahme des Drucks pro \% auch beträchtlicher; die durchschnittliche Abnahme des k.o.D. betrug 16,4 (12,9-18,4) \%und die des Drucks pro $\% 4,9(4,0-5,9) \%$.

- Nach Ablauf von 30 min näherten sich der Hämoglobin-, Eiweiss- und Fibrinogengehalt zum Anfangswert und nach $60 \mathrm{~min}$ blieben sie auf gleicher Höhe oder neigten mehr oder weniger zu Verminderung. Hingegen zeigte der k.o.D. noch einen niedrigen Wert, also erniedrigte sich auch der Druck pro \%; im Durchschnitt betrug die Abnahme des k.o.D. nach 30 min 9;0 $(6,7-12,1) \%$, nach $60 \mathrm{~min} 8,0(5,5-11,0) \%$, die des Drucks pro $\%$ nach $30 \min 3,0(1,4-5,2) \%$, nach $60 \min 2,0(1,0-4,4) \%$.

Aus obigen Resultaten kann man ersehen, dass der Einfluss der Alkalizufuhr auf das Bluteiweissbild demselben der Säurezufuhr ähnlich ist; nämlich, der k.o.D. nimmt gegenüber dem Eiweiss stärker ab, so dass sich der Druck pro \% erheblich erniedrigt, nur dass hier die Erniedrigung nicht so hochgradig wie bei Säurezufuhr ist. 


\section{Versuch an Kaninchen mit doppelseitiger Ureterenunterbindung.}

Dass bei Nierenkranken sowie bei experimentellen Nierenschädigungen die Eiweisskörper des Blutes quantitativ sowie qualitativ kedeutende Veränderungen erfahren, wobei die Erniedrigung des k.o.D. auftritt, ist bereits eine bestimmte Tatsache. Nun, es ist expẹrimentell sowie klinisch recht von grosser Bedeutung, darüber klar zu machen, wie sich die bereits durch

\section{Tabelle 4.}

Veränderungen des Bluteiweisses und dessen k.o.D. nach intravenöser Injektion von physiologischer Kochsalzlösung bei Kaninchen mit bds.

Ureterenunterbindung.

\begin{tabular}{|c|c|c|c|c|c|c|c|c|}
\hline \multirow{3}{*}{ 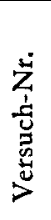 } & \multirow{3}{*}{ 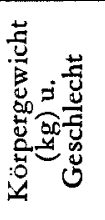 } & \multirow{3}{*}{$\begin{array}{c}\text { Zeit der } \\
\text { Blutentnahme }\end{array}$} & \multicolumn{3}{|c|}{ Hämoglobin } & \multicolumn{3}{|c|}{ Serumeiweiss } \\
\hline & & & \multirow[b]{2}{*}{$\mathrm{g} / \mathrm{dl}$} & \multicolumn{2}{|c|}{ Diff. in $\%$} & \multirow[b]{2}{*}{$\%$} & \multicolumn{2}{|c|}{ Diff. in $\%$} \\
\hline & & & & 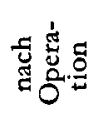 & 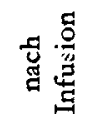 & & 吾芯苔. & 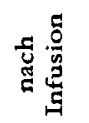 \\
\hline 1 & $\begin{array}{c}1,950 \\
1,900 \\
\delta\end{array}$ & $\begin{array}{l}\text { Anfangswert } \\
50 \text { Std. nach Operation } \\
\text { Direkt nach der Infusion } \\
30 \text { Min. " } \\
60 \text { Min. " }\end{array}$ & $\begin{array}{l}13,94 \\
11,36 \\
10,24 \\
10,89 \\
10,80\end{array}$ & $-18,5$ & $\begin{array}{l}-9,9 \\
-4,1 \\
-4,9\end{array}$ & $\begin{array}{l}5,86 \\
7,05 \\
6,45 \\
6,77 \\
6,70\end{array}$ & $+20,3$ & $\begin{array}{l}-8,5 \\
-\quad 4,0 \\
-5,0\end{array}$ \\
\hline 2 & $\begin{array}{l}1,700 \\
1,650 \\
\delta\end{array}$ & $\begin{array}{l}\text { Anfangswert } \\
50 \text { Std. nach Operation } \\
\text { Direkt nach Infusion } \\
30 \text { Min. } \\
60 \text { Min. }\end{array}$ & $\begin{array}{l}14,24 \\
11,66 \\
10,61 \\
11,11 \\
11,04\end{array}$ & $-18,1$ & $\begin{array}{l}-9,0 \\
=4,7 \\
-5,3\end{array}$ & $\begin{array}{l}5,90 \\
6,77 \\
6,16 \\
6,44 \\
6,40\end{array}$ & $+14,7$ & $\begin{array}{l}-9,0 \\
=4,9 \\
-5,5\end{array}$ \\
\hline 3 & $\begin{array}{l}1,480 \\
1,420 \\
\uparrow\end{array}$ & $\begin{array}{l}\text { Anfangswert } \\
50 \text { Std. nach Operation } \\
\text { Direkt nach Infusion } \\
30 \text { Min. } \\
60 \text { Min. }\end{array}$ & $\begin{array}{l}14,52 \\
12,20 \\
11,36 \\
11,96 \\
11,96\end{array}$ & $-16,0$ & $\begin{array}{l}-6,8 \\
=2,0 \\
-2,0\end{array}$ & $\begin{array}{l}6,49 \\
7,85 \\
7,29 \\
7,60 \\
7,55\end{array}$ & $+20,9$ & $\begin{array}{l}-7,1 \\
=3,2 \\
-\quad 3,8\end{array}$ \\
\hline 4 & $\begin{array}{l}1,800 \\
1,740 \\
\text { 今 }\end{array}$ & $\begin{array}{l}\text { Anfangswert } \\
50 \text { Std. nach Operation } \\
\text { Direkt nach Infusion } \\
30 \mathrm{Min} . " \\
60 \mathrm{Min} . "\end{array}$ & $\begin{array}{r}13,36 \\
10,80 \\
9,68 \\
10,24 \\
10,20\end{array}$ & $-19,2$ & $\begin{array}{l}-10,4 \\
-2,6 \\
-3,0\end{array}$ & $\begin{array}{l}5,38 \\
6,98 \\
6,34 \\
6,70 \\
6,66\end{array}$ & $+29,7$ & $\begin{array}{l}-9,2 \\
-4,0 \\
-4,6\end{array}$ \\
\hline 5 & $\begin{array}{r}1,700 \\
1,560 \\
.+9\end{array}$ & $\begin{array}{l}\text { Anfangswert } \\
50 \text { Std. nach Operation } \\
\text { Direkt nach Infusion } \\
30 \mathrm{Min} . \quad \\
60 \mathrm{Min} .\end{array}$ & $\begin{array}{l}13,94 \\
11,66 \\
10,52 \\
11,14 \\
11,08\end{array}$ & $-16,3$ & $\begin{array}{l}-9,8 \\
-4,4 \\
-5,0\end{array}$ & $\begin{array}{l}6,16 \\
6,92 \\
6,29 \\
6,59 \\
6,53\end{array}$ & $+12,3$ & $\begin{array}{l}-9,1 \\
=4,8 \\
-\quad 5,6\end{array}$ \\
\hline & schnitt & $\begin{array}{l}\text { Anfangswert } \\
50 \text { Std. nach Operation } \\
\text { Direkt nach Infusion } \\
30 \text { Min. " } \\
60 \text { Min. " }\end{array}$ & $\begin{array}{l}14,00 \\
11,54 \\
10,48 \\
11,12 \\
11,07\end{array}$ & $-17,6$ & $\begin{array}{l}-9,1 \\
-3,6 \\
-\quad 4,0\end{array}$ & $\begin{array}{l}5,96 \\
7,11 \\
6,51 \\
6,82 \\
6,77\end{array}$ & $+19,3$ & $\begin{array}{l}-8,4 \\
-4,1 \\
-4,8\end{array}$ \\
\hline
\end{tabular}


Funktionsausfall der Niere veränderten Bluteiweisskörper beim abnormen $\mathrm{pH}$ des Blutes, das nach intravenöser Verabreichung der Säure- bzw. Alkalilösung zustande kommt, weiter verändern.

In vorliegender Versuchsreihe wurden Kaninchen, die der doppelseitigen Ureterenunterbindung unterworfen waren, nach Ablauf von 50 Stunden nach dem Eingriff zum Versuch benutzt. Vor Vornahme der Operation wurden zuvörderst an den Tieren, welche 12 Stunden lang gehungert hatten, Bestimmungen von Hämoglobin-, Eiweiss- und Fibrinogengehalt sowie von k.o.D. und $\mathrm{pH}$ des Blutes ausgeführt. Die Bestimmungsmethoden der betreffenden Daten waren ganz dieselben, die in vorange-

Tabelle 4. Fortsetzung.

\begin{tabular}{|c|c|c|c|c|c|c|c|c|c|c|c|}
\hline \multicolumn{3}{|c|}{ K.o.D. } & \multicolumn{3}{|c|}{ Druck pro $\%$} & \multicolumn{3}{|c|}{ Fibrinogen } & \multicolumn{3}{|c|}{$\mathrm{pH}$ des Blutes } \\
\hline \multirow[b]{2}{*}{ 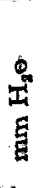 } & \multicolumn{2}{|c|}{ Diff. in \% } & \multirow[b]{2}{*}{ 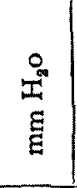 } & \multicolumn{2}{|c|}{ Dif. in $\%$} & \multirow[b]{2}{*}{$\%$} & \multicolumn{2}{|c|}{ Diff. in $\%$} & & \multicolumn{2}{|c|}{ Diff. in $\%$} \\
\hline & 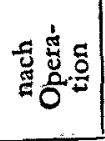 & 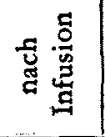 & & 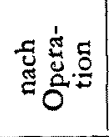 & 岳 $\frac{5}{\frac{0}{5}}$ & & 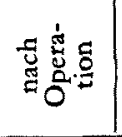 & 串 & & 焉密 & 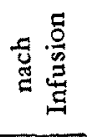 \\
\hline $\begin{array}{l}276 \\
247 \\
226 \\
238\end{array}$ & $-10,5$ & $\begin{array}{r}-8,5 \\
-3,6 \\
-5,3 \\
\end{array}$ & $\begin{array}{l}47,1 \\
35,0 \\
35,0 \\
35,1 \\
34, \\
\end{array}$ & $-25,7$ & $\begin{array}{c} \pm 0 \\
+0,3 \\
-0,3 \\
\end{array}$ & $\begin{array}{l}0,19 \\
0,56 \\
0,47 \\
0,52 \\
0,52\end{array}$ & $+194,7$ & $\begin{array}{r}-16,0 \\
-7,1 \\
-7,1 \\
\end{array}$ & $\begin{array}{l}7,35 \\
7,21 \\
7,22 \\
7,20 \\
7,19\end{array}$ & $-1,9$ & $\begin{array}{l}+0,1 \\
-0,1 \\
-0,3\end{array}$ \\
\hline $\begin{array}{l}265 \\
232 \\
211 \\
220 \\
218 \\
\end{array}$ & $-12,4$ & $\begin{array}{l}-9,0 \\
-5,2 \\
-\quad 6,0\end{array}$ & $\begin{array}{l}44,9 \\
34,3 \\
34,2 \\
34,2 \\
34,1\end{array}$ & $-23,6$ & $\begin{array}{r}-0,3 \\
-0,3 \\
-0,6 \\
\end{array}$ & $\begin{array}{l}0,21 \\
0,52 \\
0,43 \\
0,47 \\
0,47 \\
\end{array}$ & $+147,6$ & $\begin{array}{r}-17,3 \\
-9,6 \\
-9,6\end{array}$ & $\begin{array}{l}7,37 \\
7,22 \\
7,22 \\
7,22 \\
7,21 \\
\end{array}$ & $-2,0$ & $\begin{array}{l} \pm 0 \\
\pm 0 \\
-0,1\end{array}$ \\
\hline $\begin{array}{l}287 \\
257 \\
238 \\
248 \\
246\end{array}$ & $-10,4$ & $\begin{array}{l}-7,3 \\
=3,5 \\
-4,3\end{array}$ & $\begin{array}{l}44,2 \\
32,7 \\
32,6 \\
32,6 \\
32,6\end{array}$ & $-26,0$ & $\begin{array}{l}-0,3 \\
=0,3 \\
-0,3\end{array}$ & $\begin{array}{l}0,24 \\
0,56 \\
0,47 \\
0,52 \\
0,50\end{array}$ & $+133,3$ & $\begin{array}{l}-16,1 \\
-7,1 \\
-10,7\end{array}$ & $\begin{array}{l}7,32 \\
7,18 \\
7,17 \\
7,18 \\
7,17\end{array}$ & $-1,9$ & $\begin{array}{l}-0,1 \\
\pm 0 \\
-0,1\end{array}$ \\
\hline $\begin{array}{l}246 \\
216 \\
196 \\
204 \\
202\end{array}$ & $-12,2$ & $\begin{array}{l}-9,2 \\
-5,5 \\
-6,5\end{array}$ & $\begin{array}{l}45,7 \\
30,9 \\
30,9 \\
30,4 \\
30,3\end{array}$ & $-32,4$ & $\begin{array}{lc} \pm \quad 0 \\
=1,6 \\
-1,9\end{array}$ & $\begin{array}{l}0,17 \\
0,54 \\
0,45 \\
0,50 \\
0,49\end{array}$ & $+217,6$ & $\begin{array}{r}-18,5 \\
-7,4 \\
-9,2\end{array}$ & $\begin{array}{l}7,38 \\
7,14 \\
7,14 \\
7,13 \\
7,12\end{array}$ & $-3,2$ & $\begin{array}{l} \pm 0 \\
-0,1 \\
-0,3\end{array}$ \\
\hline $\begin{array}{l}280 \\
250 \\
227 \\
238 \\
235\end{array}$ & $-10,7$ & $\begin{array}{l}-9,6 \\
=4,8 \\
-6,0\end{array}$ & $\begin{array}{l}45,4 \\
36,1 \\
36,1 \\
36,1 \\
36,0\end{array}$ & $-20,5$ & $\begin{array}{cc} \pm & 0 \\
\pm & 0 \\
- & 0,3\end{array}$ & $\begin{array}{l}0,24 \\
0,75 \\
0,64 \\
0,67 \\
0,67\end{array}$ & $+212,5$ & $\begin{array}{l}-14,7 \\
-10,7 \\
-10,7\end{array}$ & $\begin{array}{l}7,35 \\
7,20 \\
7,21 \\
7,19 \\
7,17 \\
\end{array}$ & $-2,0$ & $\begin{array}{l}+0,1 \\
-0,1 \\
-0,1\end{array}$ \\
\hline $\begin{array}{l}271 \\
240 \\
220 \\
230 \\
227\end{array}$ & $-11,4$ & $\begin{array}{l}-8,3 \\
-4,2 \\
-5,4\end{array}$ & $\begin{array}{l}45,5 \\
33,8 \\
33,8 \\
33,7 \\
33,6\end{array}$ & $-25,7$ & $\begin{array}{cc} \pm & 0 \\
- & 0,3 \\
- & 0,6\end{array}$ & $\begin{array}{l}0,21 \\
0,59 \\
0,49 \\
0,54 \\
0,54\end{array}$ & $+185,9$ & $\begin{array}{r}-16,9 \\
-8,5 \\
-8,5\end{array}$ & $\begin{array}{l}7,35 \\
7,19 \\
7,19 \\
7,18 \\
7,17\end{array}$ & $-2,2$ & $\begin{array}{l} \pm 0 \\
-0,1 \\
-0,3\end{array}$ \\
\hline
\end{tabular}


hendem Kapitel ausführlich geschildert wurden. Der Grund dafür, dass beiderseitige Ureterenunterbindung als Methode zur Provokation der Funk. tionsstörung der Niere ausgewählt wurde, liegt darauf, dass die Veränderungen des Bluteiweisses bei Ureterenunterbindung unter denselben bei anderen experimentellen Nierenschädigungen die stärksten sind.

Nun wollen wir die bei den obigen Eingriffen unterworfenen Kaninchen gewonnenen Bestimmungswerte mit denen, die bei denselben Tieren im gesunden Zustand ermittelt wurden, vergleichend beobachten (Tab. 4). Der Hämoglobingehalt, der im gesunden Zustand im Mittel 14,00 g/dl betrug, nahm 50 Stunden nach erfolgter Ureterenunterbindung im Durchschnitt auf $11,54 \mathrm{~g} / \mathrm{dl}$ ab. Dagegen stieg der Serumeiweissgehalt, der sich vor dem Eingriff im Mittel 5,96\% bezifferte, 50 Stunden nach der Operation durchschnittlich auf $7,11 \%$ an, während der k.o.D., der vor der Ureterenunterbindung im Durchschnitt $271 \mathrm{~mm} \mathrm{H}_{2} \mathrm{O}$ betrug, im Mittel auf $240 \mathrm{~mm} \mathrm{H}_{2} \mathrm{O}$ abnahm, infolgedessen erniedrigte sich der Druck pro \% im Mittel von 45,5 $\mathrm{mm} \mathrm{H}_{2} \mathrm{O}$ auf $33,8 \mathrm{~mm} \mathrm{H}_{2} \mathrm{O}$.

Was den Fibrinogengehalt des Blutes betrifft, wurde von Dienst ${ }^{33)}$ bei Schwangerschaftsniere, von Kollert u. Starlinge ${ }^{38)}$, Rusznyák, Barat u. Kürthy ${ }^{39)}$ sowie Rusznyák ${ }^{409}$ bei Nephrose starke Zunahme des Plasmafibrinogens festgestellt. Oza w $\mathrm{a}^{411}$ fand bei Hunden mit Ureterenunterbindung oder Nephrektomie Zunahme des Fibrins; Tanaka ${ }^{22)}$ bei Kantharidinhunden Zunahme des Fibrinogens im Blute. In meinen Versuchen wurde auch Zunahme des Fibrinogengehaltes nachgewiesen; der Fibrinogengehalt, der vor der Ureterenunterbindung im Mittel einen Wert von $0,21 \%$ aufwies, erhöhte sich 50 Stunden nach dem Eingriff durchschnittlich auf $0,59 \%$ ， Das $\mathrm{pH}$ des Blutes, welches im gesunden Zustand im Mittel bei 7,35 gelegen war, stieg im Durchschnitt auf 7,19 ab; das Versuchstier befand sich nämlich offenbar in einem ausgesprochen azidotischen Zustand.

1. Kontrolle. Injektion von physiologischer Kochsalzlösung.

Nachdem bei 5 Kaninchen beiderseitige Ureteren unterbunden worden waren, wurde der Versuch 50 Stunden später angestellt. Zuerst wurde als Kontrolle den Kaninchen körperwarme, sterile physiologische Kochsălzlösung in Mengen von $10 \mathrm{ccm}$ pro $\mathrm{kg}$ Körpergewicht intravenös einverleibt und die analogen Daten wie in vorangehendem Versuch ermittelt. Die Ergebnisse liessen sich auf Tab. 4 zusammenfassen, in Fig. 4 sind die durchschnittlichen prozentualen Werte wiedergegeben.

Das $\mathrm{pH}$ des Blutes blieb direkt nach der Injektion von isotonischer Kochsalzlösung unverändert, aber nach 60 min neigte es mehr oder minder 
Fig. 4. Prozentuelle Blutveränderungen nach intravenöser Injektion von physiologischer Kochsalzlösung bei Kaninchen mit bds.

Ureterenunterbindung (Durchschnitt).

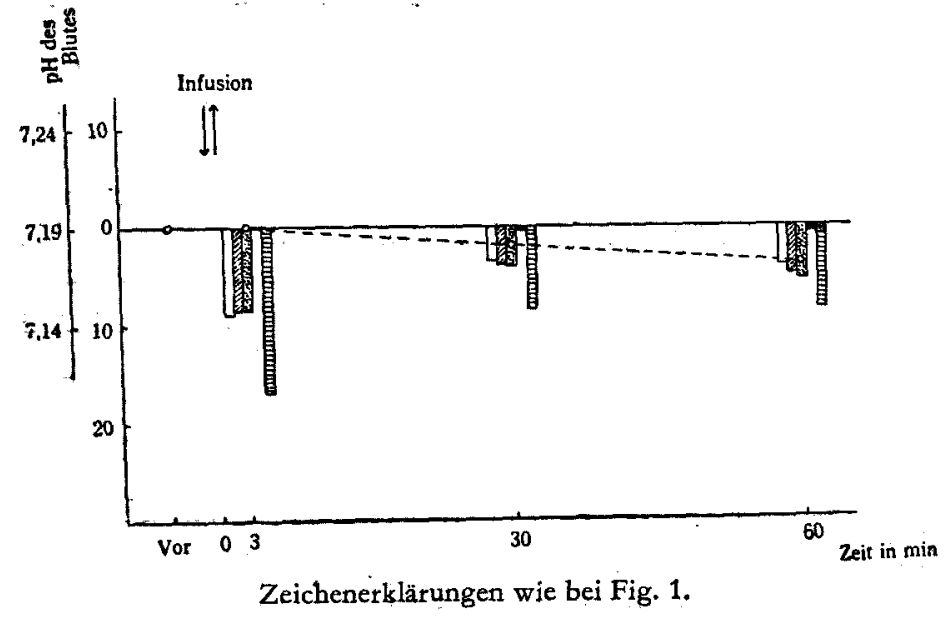

zur V̆erminderung. Im direkten Anschluss an die Injektion nahm im Mittel der Hämoglobingehalt um 9,1 $(6,8-10,4) \%$, der Eiweissgehalt um 8,4 $(7,1-9,2) \%$, der Fibrinogengehalt um 16,9 (14,7-18,5) \% und der k.o.D um $8,3(7,3-9,6) \%$ ab. Aber in darauffolgender Zeit neigten sie in allen Fällen zu Erholung; 30 min nach der Injektion näherten sie sich dem Anfangswert und nach $60 \mathrm{~min}$ blieben sie auf derselben Höhe oder stiegen sie weiter ein wenig ab. Die Sehwankungen des Eiweissgehaltes und des k.o.D. gingen stets miteinander Hand in Hand, also war der Druck pro \% nur geringen Schwankungen unterworfen.

Wenn physiologische Kochsalzlösung den Kaninchen mit beiderseitiger Ureterenunterbindung injiziert wurde, waten die Veränderungen der Hämoglobin- und Eiweisskonzentration des Blutes kleiner als die bei gesunden Tieren unter gleichem Verfahren. Dies dürfte wahrscheinlich vorwiegend auf Permeabilitätssteigerung der Kapillarwand sowie physikalisch-chemische Veränderungen des Gewebes infolge des Funktionsausfalls der Niere, zum Teil aber auch auf die Zunahme der zirkulierenden Blutmenge bei nierengeschädigten Tieren, wie Beckmann ${ }^{43)}$, Bence ${ }^{44)}$, Litzne ${ }^{45)}$, Kasiwa mura ${ }^{46)}$, A u gelin $i^{477}, \mathrm{Ok} \mathrm{a}^{48)}$ u.a. bestätigten, zurückgeführt werden.

2. Infusion von $\mathbf{N} / 10$-Salzsäurelösung.

- Es wurde 6 Kaninchen, welche 50 Stunden vor dem Versuch einer doppelseitigen. Ureterenunterbindung unterworfen waten, körperwarme N/10Salzsäurelösung, in deren Deziliter $0,9 \mathrm{~g} \mathrm{NaCl}$ aufgelöst wurde, in Mengen von $10 \mathrm{ccm}$ pro $\mathrm{kg}$ Körpergewicht intravenös verabreicht. Die Bestim- 
mungsdaten sind in Tab. 5 aufgezeichnet, Fig. 5 zeigt die prozentuale Abund Zunahme derselben im Durchschnitt.

Durch intravenöse Injektion von $\mathrm{N} / 10-\mathrm{HCl}-$ Lösung erniedrigte sich das $\mathrm{pH}$ des Blutes bei den Ureterenunterbindung unterworfenen Kaninchen

Tabelle 5 .

Veränderungen des Bluteiweisses und dessen k.o.D. nach intravenöser Injektion von N/10 Salzsäurelösung bei Kaninchen mit bds. Ureterenunterbindung.

\begin{tabular}{|c|c|c|c|c|c|c|c|c|}
\hline \multirow{3}{*}{ 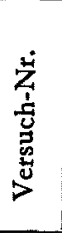 } & \multirow{3}{*}{ 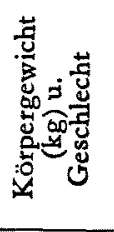 } & \multirow{3}{*}{$\begin{array}{c}\text { Zeit der } \\
\text { Blutentnahme }\end{array}$} & \multicolumn{3}{|c|}{ Hämoglobin } & \multicolumn{3}{|c|}{ Serumeiweiss } \\
\hline & & & \multirow[b]{2}{*}{$\mathrm{g} / \mathrm{dl}$} & \multicolumn{2}{|c|}{ Diff. in $\%$} & \multirow[b]{2}{*}{$\%$} & \multicolumn{2}{|c|}{ Diff. in \% } \\
\hline & & & & 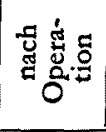 & 茑 & & 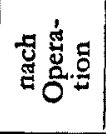 & 岳峞 \\
\hline 1 & $\begin{array}{c}1,740 \\
1,600 \\
+\end{array}$ & $\begin{array}{l}\text { Anfangswert } \\
50 \text { Std, nach Operation } \\
\text { Direkt nach Infusion } \\
30 \mathrm{Min} . \\
60 \mathrm{Min} .\end{array}$ & $\begin{array}{l}13,36 \\
10,80 \\
10,76 \\
10,52 \\
10,30\end{array}$ & $-19,2$ & $\begin{array}{r}7,6 \\
-2,6 \\
-4,6\end{array}$ & $\begin{array}{l}6,16 \\
6,92 \\
6,38 \\
6,64 \\
6,55\end{array}$ & $+12,3$ & $\begin{array}{r}-7,8 \\
-4,0 \\
-5,3 \\
\end{array}$ \\
\hline 2 & $\begin{array}{c}1,660 \\
1,530 \\
8\end{array}$ & $\begin{array}{l}\text { Anfangswert } \\
50 \text { Std. nach Operation } \\
\text { Direkt nach Infusion } \\
30 \text { Min. } \\
60 \text { Min. }\end{array}$ & $\begin{array}{r}14,24 \\
10,52 \\
9,86 \\
10,20 \\
10,20\end{array}$ & $-26,1$ & $\begin{array}{r}-6,3 \\
-3,0 \\
+3,0 \\
\end{array}$ & $\begin{array}{l}5,72 \\
7,16 \\
6,73 \\
6,90 \\
6,90\end{array}$ & $+25,2$ & $\begin{array}{l}-6,0 \\
=3,6 \\
-3,6 \\
\end{array}$ \\
\hline 3 & $\begin{array}{c}1,800 \\
1,650 \\
\delta\end{array}$ & $\begin{array}{l}\text { Anfangswert } \\
50 \text { Std. nach Operation } \\
\text { Direkt nach Infusion } \\
30 \mathrm{Min} . " \\
60 \mathrm{Min} . "\end{array}$ & $\begin{array}{l}14,80 \\
12,80 \\
11,96 \\
12,24 \\
12,24\end{array}$ & $-13,5$ & $\begin{array}{r}-6,3 \\
-4,4 \\
-4,4\end{array}$ & $\begin{array}{l}5,99 \\
7,55 \\
7,07 \\
7,33 \\
7,05\end{array}$ & $+26,0$ & $\begin{array}{l}-6,3 \\
-2,9 \\
-6,6\end{array}$ \\
\hline 4 & $\begin{array}{l}1,740 \\
1,710\end{array}$ & $\begin{array}{l}\text { Anfangswert } \\
50 \text { Std. nach Operation } \\
\text { Direkt nach Infusion } \\
30 \text { Min. } \\
60 \text { Min. " }\end{array}$ & $\begin{array}{l}14,52 \\
12,20 \\
11,36 \\
11,96 \\
11,96\end{array}$ & $-15,7$ & $\begin{array}{r}-6,7 \\
-2,3 \\
-2,3 \\
\end{array}$ & $\begin{array}{l}6,49 \\
7,85 \\
7,42 \\
7,63 \\
7,55\end{array}$ & $+20,9$ & $\begin{array}{r}-5,5 \\
-2,8 \\
-3,8 \\
\end{array}$ \\
\hline 5 & $\begin{array}{c}2,380 \\
2,240 \\
\delta\end{array}$ & $\begin{array}{l}\text { Anfangswert } \\
50 \text { Std. nach Operation } \\
\text { Direkt nach Infusion } \\
30 \mathrm{Min} . \\
60 \mathrm{Min} .\end{array}$ & $\begin{array}{l}14,24 \\
12,80 \\
11,20 \\
12,24 \\
11,96\end{array}$ & $-10,1$ & $\begin{array}{r}-12,5 \\
-5,1 \\
-6,6 \\
\end{array}$ & $\begin{array}{l}5,64 \\
6,98 \\
6,16 \\
6,59 \\
6,51\end{array}$ & $+23,7$ & $\begin{array}{r}-11,7 \\
-5,6 \\
-\quad 6,7 \\
\end{array}$ \\
\hline 6 & $\begin{array}{c}1,680 \\
1,500 \\
6\end{array}$ & $\begin{array}{l}\text { Anfangswert } \\
50 \text { Std. nach Operation } \\
\text { Direkt nach Infusion } \\
30 \mathrm{Min} . " \\
60 \mathrm{Min} . "\end{array}$ & $\begin{array}{r}13,36 \\
10,80 \\
9,80 \\
10,52 \\
10,52\end{array}$ & $-19,2$ & $\begin{array}{r}-9,2 \\
-2,6 \\
-2,6 \\
\end{array}$ & $\begin{array}{l}5,38 \\
6,98 \\
6,34 \\
6,77 \\
6,66 \\
\end{array}$ & 429,7 & $\begin{array}{r}-9,2 \\
-\quad 3,0 \\
-\quad 4,6 \\
\end{array}$ \\
\hline $\mathrm{Du}$ & schnitt & $\begin{array}{l}\text { Anfangswert } \\
50 \text { Std. nach Operation } \\
\text { Direkt nach Infusion } \\
30 \mathrm{Min} \text {. " } \\
60 \mathrm{Min} . "\end{array}$ & $\begin{array}{l}14,09 \\
11,65 \\
10,82 \\
11,28 \\
11,19\end{array}$ & $-17,3$ & $\begin{array}{l}-7,1 \\
-3,2 \\
-3,9\end{array}$ & $\begin{array}{l}5,90 \\
7,24 \\
6,68 \\
6,98 \\
6,87\end{array}$ & $+18,5$ & $\begin{array}{l}-7,7 \\
=3,6 \\
=5,1\end{array}$ \\
\hline
\end{tabular}


stärker und langdauernder als bei normalen Kaninchen, sogar selbst nach Ablauf von 60 min kehrte es noch nicht zum Anfangswert zurück.

Unmittelbar nach der Injektion nahm durchschnittlich der Hämoglobingehalt um 7,1 (6,3-12,5) \%, der Eiweissgehalt um 7,7 (5,5-11,7) \% und det Fibrinogengehalt um 14,5 (12,2-19,2) \% ab; diese Abnahmen erwiesen sich etwas geringer als die im Kontrollversuch. Im Gegensatz dazu zeigte

Tabelle 5. Fortsetzung.

\begin{tabular}{|c|c|c|c|c|c|c|c|c|c|c|c|}
\hline \multicolumn{3}{|c|}{ K.o. D. } & \multicolumn{3}{|c|}{ Druck pro $\%$} & \multicolumn{3}{|c|}{ Fibrinogen } & \multicolumn{3}{|c|}{$\mathrm{pH}$ des Blutes } \\
\hline \multirow[b]{2}{*}{$\begin{array}{l}0 \\
0 \\
\underline{z} \\
\xi\end{array}$} & \multicolumn{2}{|c|}{ Diff. in $\%$} & \multirow[b]{2}{*}{ 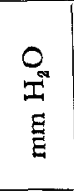 } & \multicolumn{2}{|c|}{ Diff. in \% } & \multirow[b]{2}{*}{$\%$} & \multicolumn{2}{|c|}{ Diff. in $\%$} & & \multicolumn{2}{|c|}{ Diff. in $\%$} \\
\hline & 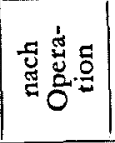 & 乐 $\frac{c}{\frac{c}{0}}$ & & 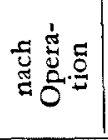 & 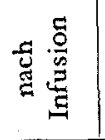 & & 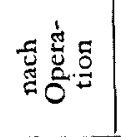 & 点 & & 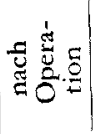 & 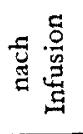 \\
\hline $\begin{array}{l}280 \\
244 \\
205 \\
216 \\
218\end{array}$ & $-12,8$ & $\begin{array}{l}-16,0 \\
-11,5 \\
-10,6\end{array}$ & $\begin{array}{l}45,4 \\
35,3 \\
32,1 \\
32,5 \\
33,3\end{array}$ & $-22,2$ & $\begin{array}{l}-10,0 \\
-8,6 \\
-5,7\end{array}$ & $\begin{array}{l}0,24 \\
0,56 \\
0,49 \\
0,56 \\
0,52\end{array}$ & $+133,3$ & $\begin{array}{c}-12,5 \\
\pm 0 \\
\pm 7,1\end{array}$ & \begin{tabular}{l|}
7,33 \\
7,23 \\
7,12 \\
7,15 \\
7,17
\end{tabular} & $-1,4$ & $\begin{array}{l}-1,5 \\
-1,1 \\
-0,8 \\
\end{array}$ \\
\hline $\begin{array}{l}279 \\
256 \\
210 \\
220 \\
224\end{array}$ & $-8,2$ & $\begin{array}{l}-18,0 \\
-14,0 \\
-12,5\end{array}$ & $\begin{array}{l}48,8 \\
35,7 \\
31,2 \\
31,9 \\
32,5\end{array}$ & $-26,8$ & $\begin{array}{r}-14,4 \\
-11,9 \\
-9,8\end{array}$ & \begin{tabular}{l|}
0,21 \\
0,49 \\
0,43 \\
0,49 \\
0,45
\end{tabular} & $+133,3$ & $\begin{array}{c}-12,2 \\
\pm \quad 0 \\
-8,2\end{array}$ & $\begin{array}{l}7,35 \\
7,19 \\
7,06 \\
7,14 \\
7,14 \\
\end{array}$ & $-2,2$ & $\begin{array}{l}-1,8 \\
-0,7 \\
-0,7 \\
\end{array}$ \\
\hline $\begin{array}{l}277 \\
250 \\
198 \\
219 \\
216 \\
\end{array}$ & $-9,7$ & $\begin{array}{l}-20,8 \\
-12,4 \\
-13,6 \\
\end{array}$ & $\begin{array}{l}46,2 \\
33,1 \\
28,0 \\
29,9 \\
30,6\end{array}$ & $-28,3$ & $\begin{array}{r}-15,4 \\
-9,7 \\
-7,5 \\
\end{array}$ & $\begin{array}{l}0,19 \\
0,69 \\
0,60 \\
0,71 \\
0,65\end{array}$ & $+263,2$ & $\begin{array}{r}-13,0 \\
+\quad 2,9 \\
-\quad 5,8 \\
\end{array}$ & $\begin{array}{l}7,41 \\
7,23 \\
7,14 \\
7,17 \\
7,19\end{array}$ & $-2,4$ & $\begin{array}{l}-1,2 \\
-0,8 \\
-0,5 \\
\end{array}$ \\
\hline $\begin{array}{l}287 \\
254 \\
212 \\
224 \\
224\end{array}$ & $-11,5$ & $\begin{array}{l}-16,5 \\
-11,8 \\
-11,8 \\
\end{array}$ & $\begin{array}{l}44,2 \\
32,3 \\
28,6 \\
29,3 \\
29,7\end{array}$ & $-26,9$ & $\begin{array}{r}-11,4 \\
-9,3 \\
-8,0 \\
\end{array}$ & $\begin{array}{l}0,24 \\
0,56 \\
0,47 \\
0,56 \\
0,52\end{array}$ & $+133,3$ & $\begin{array}{l}-16,1 \\
\pm 0 \\
=7,1\end{array}$ & $\begin{array}{l}7,36 \\
7,18 \\
7,09 \\
7,14 \\
7,14\end{array}$ & $-2,4$ & $\begin{array}{l}-1,2 \\
-0,5 \\
-0,5 \\
\end{array}$ \\
\hline $\begin{array}{l}264 \\
232 \\
180 \\
200 \\
198 \\
\end{array}$ & $-12,1$ & $\begin{array}{l}-22,4 \\
-13,8 \\
-14,6\end{array}$ & \begin{tabular}{|l|}
46,8 \\
33,2 \\
29,2 \\
30,3 \\
30,4
\end{tabular} & $-29,0$ & $\begin{array}{r}-12,0 \\
-8,7 \\
-8,4\end{array}$ & $\begin{array}{l}0,19 \\
0,52 \\
0,42 \\
0,49 \\
0,45\end{array}$ & $+173,7$ & $\begin{array}{r}-19,2 \\
-5,8 \\
-13,5\end{array}$ & $\begin{array}{l}0,32 \\
7,18 \\
7,08 \\
7,12 \\
715\end{array}$ & $-1,9$ & $\begin{array}{l}-1,4 \\
-0,8 \\
-0,4\end{array}$ \\
\hline $\begin{array}{l}250 \\
225 \\
174 \\
198 \\
196\end{array}$ & $-10,0$ & $\begin{array}{l}-22,7 \\
-16,4 \\
-12,8\end{array}$ & $\begin{array}{l}46,5 \\
32,2 \\
27,4 \\
29,2 \\
29,4\end{array}$ & $-28,6$ & $\begin{array}{r}-14,9 \\
-9,3 \\
-8,7\end{array}$ & $\begin{array}{l}0,21 \\
0,49 \\
0,42 \\
0,52 \\
0,47\end{array}$ & $+133,3$ & $\begin{array}{r}-14,3 \\
+\quad 6,1 \\
-4,1\end{array}$ & $\begin{array}{l}7,31 \\
7,20 \\
7,04 \\
7,12 \\
7,14\end{array}$ & $-1,5$ & $\begin{array}{l}-2,2 \\
-1,1 \\
-0,8 \\
\end{array}$ \\
\hline $\begin{array}{l}273 \\
243 \\
196 \\
213 \\
213\end{array}$ & $-11,0$ & $\begin{array}{l}-19,3 \\
-12,3 \\
-12,3\end{array}$ & $\begin{array}{l}46,3 \\
33,6 \\
29,4 \\
30,5 \\
31,0\end{array}$ & $-27,4$ & $\begin{array}{r}-12,5 \\
-9,2 \\
-7,7\end{array}$ & $\begin{array}{l}0,21 \\
0,55 \\
0,47 \\
0,55 \\
0,51\end{array}$ & $+161,9$ & $\begin{array}{l}-14,5 \\
\pm 0 \\
-7,3\end{array}$ & $\begin{array}{l}7,35 \\
7,20 \\
7,09 \\
7,14 \\
7,15\end{array}$ & $-2,1$ & $\begin{array}{l}-1,5 \\
-0,8 \\
-0,7\end{array}$ \\
\hline
\end{tabular}


Fig. 5. Prozentuelle Blutveränderungen nach intravenöser Injektion von $\mathrm{N} / 10^{-\mathrm{HCl}-L o ̈ s u n g}$ bei Kaninchen mit bds. Ureterenunterbindung (Durchschnitt).

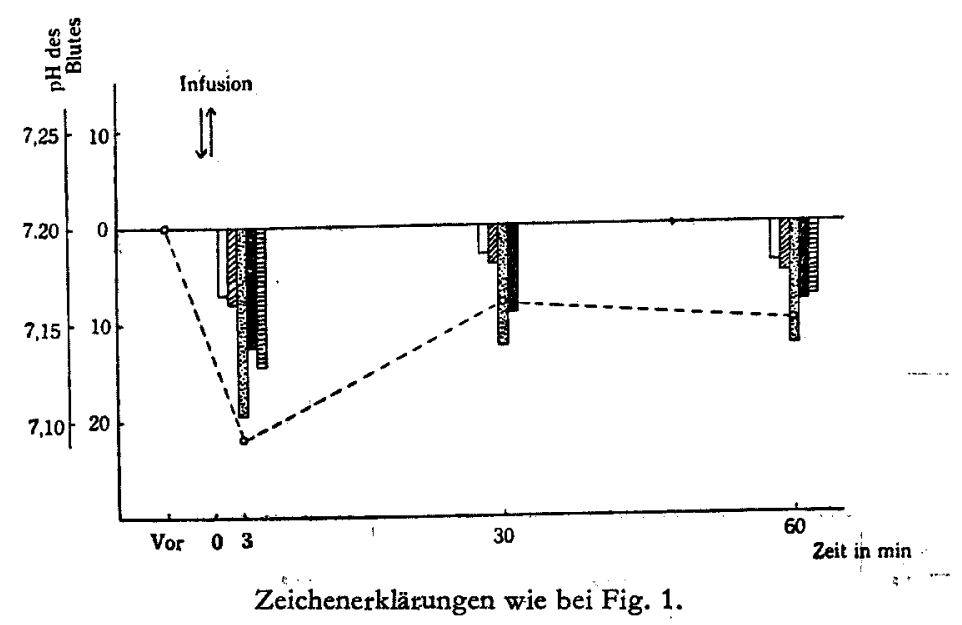

der k.o.D. eine bettächtliche Abnahme, welche im Durchschnitt ja einen Wert von 19,3 (16,0-22,7) \% aufwies, demgemäss erfolgte die Erniedrigung des Drucks pro \% auch stärker; die Abnahme belief sich im Mitel auf 12,5 (10,015,4) \%. 30 min nach der Injektion näherten sich der Hämoglobin- u. Eiweissgehalt dem früheren Niveau. Der Fibrinogenwert kehrt sich in 3 Versuchen (Versuch 1, 2 u. 4) zum Anfangswert zurück, in anderen 2 Versuchen (Versuch 3 u. 6) überschritt er den Vorwert, während er im übrigen einen Versuch (Versuch 5) sich etwas niedriger als der Vorwert erwies; nach einzelnen Fällen betrachtet, fällt die Wiederherstellung des Fibrinogengehaltes besonders ausgeprägt in die Augen. Der k.o.D. zeigte dagegen noch eine ziemliche Abnahme, welche im Mittel 12,3 (11,5-16,5) \% betrug und der Druck pro \% setzte sich im Durchschnitt um $9,2(8,6-11,9) \%$ herab. Nach 60 min erlitten der Hämoglobin-, Eiweiss- und Fibtinogenwert keine bemerkbaren Schwankungen und der k.o.D. blieb dagegen auf niedrigere Höhe, folglich zeigte der Druck pro \% auch noch eine Abnahme von durchschnittlich $7,7(5,7-9,8) \%$.

Kürzum, wie es an Ta.b 5 und Fig. 5 erkeñnbar ist, wär die durch dië Injektion von N/10-HCl-Lösung hervorgerufene Senkung von pH des Blutes bei Kaninchen mit unterbundenen Ureteren stärker und langdauernder als dieselbe bei normalen Kaninchen und die Enniedrigung des k,o.D. erfolgte auch gegenüber der Kontrolle beträchtlicher und langdauernder.

3. Injektion von isotonischer Alkalipuffertösung.

In vorliegender Versuchsreihe wurde 5 Kaninchen, welche doppel- 
seitiger Ureterenunterbindung unterworfen waren, isotonische Alkalipufferlösung ( $\mathrm{pH}=9,30)$ in Mengen von $10 \mathrm{ccm}$ pro $\mathrm{kg}$ Körpergewicht intravenös injiziert: Die Versuchsergebnisse sind auf Tab. 6 und Fig. 6 dargestellt.

Das $\mathrm{pH}$ des Blutes nahm direkt nach der Injektion vorübergehend $z \mathrm{u}$, aber nach Ablauf von 30 min kehrte es fast zum Vorwert zurück.

Unmittelbar nach der Injektion nahm der Hämoglobingehalt im Mittel um 8,7 (6,1-11,5) \%, der Eiweissgehalt um 8,6 (6,1-12,2) \%, der Fibrinogen-

Tabelle 6.

Veränderungen des Bluteiweisses und dessen k.o.D. nach intravenöser Injektion von isotonischer Alkalipufferlösung $(\mathrm{pH}=9,30)$ bei Kaninchen mit bds. Ureterenunterbindung.

\begin{tabular}{|c|c|c|c|c|c|c|c|c|}
\hline \multirow{3}{*}{ 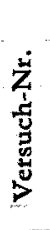 } & \multirow{3}{*}{ 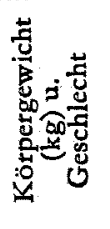 } & \multirow{3}{*}{$\begin{array}{c}\text { Zeit der } \\
\text { Blutentnahme }\end{array}$} & \multicolumn{3}{|c|}{ Hämoglobin } & \multicolumn{3}{|c|}{ Serumeiweiss } \\
\hline & & & \multirow[b]{2}{*}{$\mathrm{g} / \mathrm{dl}$} & \multicolumn{2}{|c|}{ Diff. in \% } & \multirow[b]{2}{*}{$\%$} & \multicolumn{2}{|c|}{ Diff. in \% } \\
\hline & & & & 돈 & 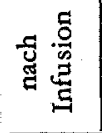 & & 宽 & 岳 \\
\hline 1 & $\begin{array}{c}1,600 \\
1,510 \\
8\end{array}$ & $\begin{array}{l}\text { Anfangswert } \\
50 \text { Std. nach Operation } \\
\text { Direkt nach Infusion } \\
30 \mathrm{Min} . " \\
60 \mathrm{Min} . "\end{array}$ & $\begin{array}{l}13,94 \\
11,66 \\
10,64 \\
11,08 \\
10,80\end{array}$ & $-16,3$ & $\begin{array}{l}-8,7 \\
-5,0 \\
-7,4\end{array}$ & $\begin{array}{l}5,86 \\
6,77 \\
6,16 \\
6,42 \\
6,19\end{array}$ & $+15,5$ & $\begin{array}{r}-9,0 \\
-5,2 \\
-8,6\end{array}$ \\
\hline 2 & $\begin{array}{c}1,500 \\
1,450 \\
\text { 우 }\end{array}$ & $\begin{array}{l}\text { Anfangswert } \\
50 \mathrm{Std} \text {. nach Operation } \\
\text { Direkt nach Infusion } \\
30 \mathrm{Min} . " \\
60 \mathrm{Min} . "\end{array}$ & $\begin{array}{r}13,94 \\
11,08 \\
9,80 \\
10,52 \\
10,52\end{array}$ & $-20,5$ & $\begin{array}{r}-11,5 \\
-5,0 \\
-5,0 \\
\end{array}$ & $\begin{array}{l}5,79 \\
6,97 \\
6,12 \\
6,55 \\
6,55\end{array}$ & $+20,4$ & $\begin{array}{l}-12,2 \\
-6,0 \\
-\quad 6,0 \\
\end{array}$ \\
\hline 3 & $\begin{array}{c}1,550 \\
1,410 \\
\text { 우 }\end{array}$ & $\begin{array}{l}\text { Anfangswert } \\
50 \text { Std. nach Operation } \\
\text { Direkt nach Infusion } \\
30 \mathrm{Min} . " \\
60 \mathrm{Min} . "\end{array}$ & $\begin{array}{l}14,52 \\
12,24 \\
11,36 \\
11,96 \\
11,96\end{array}$ & $-15,7$ & $\begin{array}{l}-7,2 \\
-\quad 2,2 \\
-\quad 2,2\end{array}$ & $\begin{array}{l}5,83 \\
7,05 \\
6,62 \\
6,88 \\
6,95\end{array}$ & $+20,9$ & $\begin{array}{l}-6,1 \\
-2,4 \\
-1,4\end{array}$ \\
\hline 4 & $\begin{array}{c}1,500 \\
1,400 \\
8\end{array}$ & $\begin{array}{l}\text { Anfangswert } \\
50 \text { Std. nach Operation } \\
\text { Direkt nach Infusion } \\
30 \text { Min. " } \\
60 \text { Min. " }\end{array}$ & $\begin{array}{l}14,24 \\
11,96 \\
10,80 \\
11,36 \\
11,32\end{array}$ & $-16,0$ & $\begin{array}{l}-9,7 \\
-5,0 \\
-5,3\end{array}$ & $\begin{array}{l}5,90 \\
6,90 \\
6,30 \\
8,47 \\
6,42\end{array}$ & $+16,9$ & $\begin{array}{r}-8,7 \\
-6,2 \\
-\quad 6,9 \\
\end{array}$ \\
\hline 5 & $\begin{array}{c}1,600 \\
1,540 \\
\delta\end{array}$ & $\begin{array}{l}\text { Anfangswert } \\
50 \text { Std. nach Operation } \\
\text { Direkt nach Infusion } \\
30 \mathrm{Min} . \\
60 \mathrm{Min} .\end{array}$ & $\begin{array}{l}13,94 \\
11,36 \\
10,67 \\
10,98 \\
10,95\end{array}$ & $-18,5$ & $\begin{array}{r}-6,1 \\
-3,3 \\
-3,6\end{array}$ & $\begin{array}{l}5,85 \\
6,95 \\
6,47 \\
6,73 \\
6,68\end{array}$ & $+18,8$ & $\begin{array}{l}-6,9 \\
-3,2 \\
-3,9\end{array}$ \\
\hline & chnitt & $\begin{array}{l}\text { Anfangswert } \\
50 \text { Std. nach Operation } \\
\text { Direkt nach Infusion } \\
30 \mathrm{Min} . \\
60 \mathrm{Min} .\end{array}$ & $\begin{array}{l}14,12 \\
11,16 \\
10,65 \\
11,18 \\
11,11\end{array}$ & $-17,4$ & $\begin{array}{l}-8,7 \\
-4,3 \\
-4,7\end{array}$ & $\begin{array}{l}5,85 \\
6,93 \\
6,33 \\
6,61 \\
6,56\end{array}$ & $+18,5$ & $\begin{array}{l}-8,6 \\
-4,6 \\
-5,3\end{array}$ \\
\hline
\end{tabular}


gehalt um 16, $7(13,0-20,4) \%$ und der k.o.D. um 8,7 (5,2-12,0) \% ab. $\mathrm{Da}_{2}$ sich das Eiweiss, wie es an obengenannten Zifferzahlen erkennbar ist, parallel mit dem k.o.D. veränderte, blieb der Druck pro \% fást unverändert.

Was weitere Schwankungen dieser Daten anbetrifft, hatten das Hämoglo. bin, Eiweiss sowie der Fibrinogen nach $30 \mathrm{~min}$ Neigung sich zu erholen und nach $60 \mathrm{~min}$ nahmen sie wieder mehr oder weniger ab. Der k:o.D. erholte sich auch augenfällig und da diese Wiederherstellung bei weitem beträchtlicher als die des' Eiweisses war, überschritt der Druck pro $\%$ den Vorwert; diese Zunahme wurde im Mittel zu 3,9 (2,4-5,3) \% ermittelt. Nach 60 min erfuhr der k.o.D. mehr oder minder leichte Abnahme, aber der

Tabelle 6. Fortsetzung.

\begin{tabular}{|c|c|c|c|c|c|c|c|c|c|c|c|}
\hline \multicolumn{3}{|c|}{ K.o. D. } & \multicolumn{3}{|c|}{ Druck pro \% } & \multicolumn{3}{|c|}{ Fibrinogen } & \multicolumn{3}{|c|}{ pH des Blutes } \\
\hline \multirow[b]{2}{*}{ 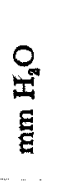 } & \multicolumn{2}{|c|}{ Diff. in \% } & \multirow[b]{2}{*}{$\begin{array}{l}0 \\
\text { 戛 }\end{array}$} & \multicolumn{2}{|c|}{ Diff, in \% } & \multirow[b]{2}{*}{$\%$} & \multicolumn{2}{|c|}{ Diff. in \% } & & \multicolumn{2}{|c|}{ Diff. in $\%$} \\
\hline & 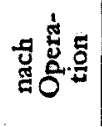 & 氬 & & 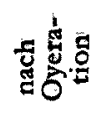 & 胥兽 & & 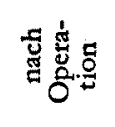 & 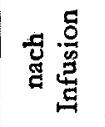 & & 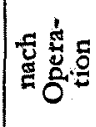 & 总 \\
\hline $\begin{array}{l}262 \\
226 \\
204 \\
225 \\
210\end{array}$ & $-13,7$ & $\begin{array}{r}-10,6 \\
-0,4 \\
-7,0 \\
\end{array}$ & $\begin{array}{l}44,7 \\
33,4 \\
33,3 \\
35,0 \\
33,9\end{array}$ & $+25,3$ & $\begin{array}{r}-0,3 \\
+4,8 \\
+1,6\end{array}$ & $\begin{array}{l}0,24 \\
0,75 \\
0,64 \\
0,69 \\
0,67\end{array}$ & $+212,5$ & $\begin{array}{r}-14,7 \\
-8,0 \\
-10,7\end{array}$ & $\begin{array}{l}7,37 \\
7,22 \\
7,27 \\
7,24 \\
7,22\end{array}$ & $-2,0$ & $\begin{array}{l}+0,7 \\
+0,3 \\
\pm 0\end{array}$ \\
\hline $\begin{array}{l}267 \\
234 \\
206 \\
228 \\
222\end{array}$ & $-12,3$ & $\begin{array}{r}-12,0 \\
-2,6 \\
-5,1 \\
\end{array}$ & $\begin{array}{l}46,1 \\
33,6 \\
33,7 \\
34,8 \\
33,9\end{array}$ & $-27,1$ & $\begin{array}{l}+0,3 \\
+\quad 3,6 \\
+0,9\end{array}$ & $\begin{array}{l}0,19 \\
0,49 \\
0,39 \\
0,43 \\
0,43\end{array}$ & $+154,9$ & $\begin{array}{l}-20,4 \\
-12,2 \\
-12,2\end{array}$ & $\begin{array}{l}7,37 \\
7,21 \\
7,26 \\
7,21 \\
7,19\end{array}$ & $-2,2$ & $\begin{array}{l} \pm 0,7 \\
\pm 0 \\
-0,3\end{array}$ \\
\hline $\begin{array}{l}254 \\
230 \\
218 \\
230 \\
228\end{array}$ & $-9,4$ & $\begin{array}{l}-5,2 \\
\pm \quad 0 \\
=0,9\end{array}$ & $\begin{array}{l}43,6 \\
32,6 \\
32,9 \\
33,4 \\
32,8\end{array}$ & $-25,2$ & $\begin{array}{l}+0,9 \\
+2,4 \\
+0,6\end{array}$ & $\begin{array}{l}0,17 \\
0,54 \\
0,45 \\
0,49 \\
0,47\end{array}$ & $+217,6$ & $\begin{array}{l}-16,7 \\
-9,2 \\
-13,0\end{array}$ & $\begin{array}{l}7,38 \\
7,14 \\
7,21 \\
7,16 \\
7,14\end{array}$ & $-3,2$ & $\begin{array}{l}+1,0 \\
+0,3 \\
\pm 0\end{array}$ \\
\hline $\begin{array}{l}257 \\
223 \\
202 \\
220 \\
212 \\
\end{array}$ & $-13,2$ & $\begin{array}{r}-9,4 \\
-1,3 \\
-\quad 4,9 \\
\end{array}$ & $\begin{array}{l}43,5 \\
32,3 \\
32,1 \\
34,0 \\
33,0\end{array}$ & $-25,7$ & $\begin{array}{l}-0,6 \\
+5,3 \\
+\quad 2,2\end{array}$ & $\begin{array}{l}0,21 \\
0,52 \\
0,43 \\
0,47 \\
0,45\end{array}$ & $+147,6$ & $\begin{array}{l}-17,3 \\
-9,6 \\
-13,5\end{array}$ & $\begin{array}{l}7,35 \\
7,21 \\
7,27 \\
7,22 \\
7,21\end{array}$ & $-3,3$ & $\begin{array}{l}+0,8 \\
+0,1 \\
\pm 0\end{array}$ \\
\hline $\begin{array}{l}265 \\
235 \\
219 \\
235 \\
232\end{array}$ & $-11,3$ & $\begin{array}{l}-6,8 \\
\pm \quad 0 \\
\pm 1,3 \\
\end{array}$ & $\begin{array}{l}45,3 \\
33,8 \\
33,9 \\
34,9 \\
34,7\end{array}$ & $-25,4$ & $\begin{array}{l}+0,3 \\
+3,2 \\
+2,7 \\
\end{array}$ & $\begin{array}{l}0,17 \\
0,67 \\
0,60 \\
0,64 \\
0,64\end{array}$ & $+305,9$ & $\begin{array}{r}-13,0 \\
-7,2 \\
-7,2\end{array}$ & $\begin{array}{l}7,31 \\
7,18 \\
7,24 \\
7,18 \\
7,16\end{array}$ & $-1,8$ & $\begin{array}{l}+0,8 \\
\pm 0 \\
-0,3\end{array}$ \\
\hline $\begin{array}{l}261 \\
230 \\
210 \\
228 \\
221\end{array}$ & $-11,9$ & $\begin{array}{l}-8,7 \\
=0,9 \\
-3,9\end{array}$ & $\begin{array}{l}44,6 \\
33,1 \\
33,2 \\
33,4 \\
33,7\end{array}$ & $-25,8$ & $\begin{array}{l}\cdot \\
+\quad 0,3 \\
+3,9 \\
+1,8\end{array}$ & $\begin{array}{l}0,20 \\
0,60 \\
0,50 \\
0,54 \\
0,53\end{array}$ & $+200,0$ & $\begin{array}{l}-16,7 \\
-10,0 \\
-11,7\end{array}$ & $\begin{array}{l}7,36 \\
7,19 \\
7,25 \\
7,20 \\
7,18\end{array}$ & $-2,3$ & $\begin{array}{r}+0,8 \\
+0,1 \\
-0,1\end{array}$ \\
\hline
\end{tabular}


Druck pro \% blieb noch etwas über dem Anfangsniveau.

Kurz zusammengefasst, erweist sich der Einfluss der Alkalizufuhr auf das $\mathrm{pH}$ des Blutes bei Kaninchen mit unterbundenen Ureteren geringer als bei gesunden Kaninchen und die Wiederherstellung des einmal abgesunkenen k.o.D. erfolgt schneller als die des Eiweisses, was mit dem Anstieg des Drucks pro $\%$ einhergehrt.

Fig. 6. Prozentuelle Blutveränderungen nach intravenöser Injektion von isotonischer Alkalipufferlösung ( $\mathrm{pH}=9,30)$ bei Kaninchen mit bds. Ureterenunterbindung (Durchschnitt).

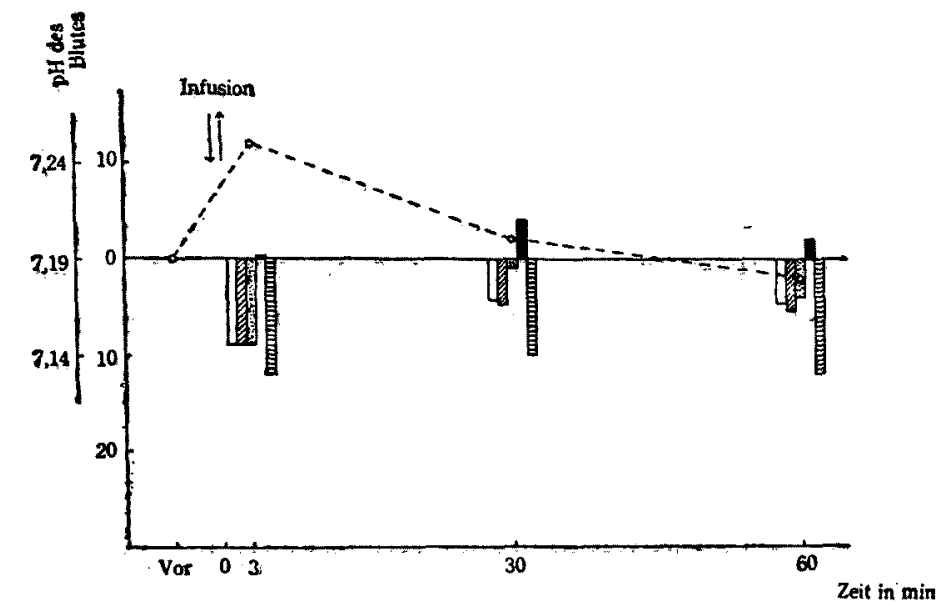

Zeichenerklärungen wie bei Fig. 1.

\section{Übersicht der Versuchsergebnisse und Besprechung.}

Obige Daten lassen sich kurz nochmals wie folgt zusammenfassen: Wird bei normalen Kaninchen die H-Ionenkonzentration des Blutes durch intravenöse Injektion von Säure- bzw. Alkalilösung verändert, so kommt die Verdünnung des Blutes etwas geringer als bei der Injektion von physiologi. scher Kochsalzlösung gleicher Menge zustande, hingegen nimmt der k.o.D. des Blutes bei Säure- bzw. Alkalizufuhr beträchtlicher ab, also tritt die Erniedrigung des Drucks pro .\% stärker als beim Kontrollversuch auf. Bei den durch doppelseitige Uretrenunterbindung urämisch gewordenen Tieren hat der Einfluss der Säurezufuhr auf das Bluteiweiss und dessen k.o.D. gleiche Neigung wie bei normalen Kaninchen, aber er ist doch an Grad offenbar erheblicher, während die Alkalizaufuhr auf die Eiweisskörper des Blutes anders als bei normalen Kaninchen einwirkt; hierbei halten die Schwankungen des k.o.D. mit denen des Bluteiweisses fast gleichen Schritt, aber die Wieder- 
herstellung des k.o:D. auf den Vorwert erfolgt schneller als die des Bluteiweisses, so dass sich der Druck pro \% erhöht.

Aus den Versuchsergebnissen der eingangs angeführten Autoren ist es exsichtlich, dass der extravale Austritt der Blutflüssigkeit bei intravenöser Injektion von Säure- bzw. Alkalilösung gesteigert sei als bei intravenöser Verabreichung von physiologischer Kochsalzlösung. In vorliegenden Versuchen an gesunden Kaninchen erfolgt die Abnahme der Hämoglobinkonzentration bei Säure- bzw. Alkalizufuhr geringer als beim Kontrollversuch; nämlich der Verdünnungsgrad des Blutes bei ersteren ist geringer. Mit anderen Worten, findet der extravasale Austritt der Blutflüssigkeit bei Säurebzw. Alkalizufuhr in höherem Masse als im Kontrollversuch statt, wa's man daraus verstehen kann, dass bei Azidosis sowie Alkalosis die Permeabilitätssteigerung der Gefässwand und die erhöhte Quellbrakeit des Gewebes vorkommen. $D_{a}$ die Schwankungen des Hämoglobin- und Eiweissgehaltes stets miteinander Hand in Hand gehen, möchten die Veränderungen des letzteren auf den ersten Blick für eine Folge einfacher Blutverdünnung gehalten werden. Aber, aus den Veränderungen des k.o.D. und Fibrinogens kann man mit Recht schliessen, dass es nicht auf einfache Blutverdünnung beruht; bei Säure- bzw. Alkalizufuhr sinkt der k.o.D. erheblicher als der Eiweissgehalt, weshalb die Abnahme des Drucks pro \% ausgeprägt vor sich geht. Ausserdem erfolgt die Abnahme des Fibrinogengehaltes hier auffallend kleiner als bei der Infektion von physiologischer Kochsalzlösung. Eben genannte Vetänderungen weisen darauf hin, dass sich das Bluteiweiss bei Säure- bzw. Alkalizufuhr nicht nur quantitativ, sondern auch qualitativ verändert, wobei die Molekularaggregate der Bluteiweisskörper nach grobdisperser Seite hin verschoben werden. Diese Veränderung dürfte einerseits darauf beruhen, dass bei Säure- bzw. Alkalizufuhr kleine Eiweissteilchen wie Albuminarten im Blute durch die Gefässwand mit gesteigerter Permeabilität hindurch reichlich in das Gewebe in der Weise „Albuminurie ins Gewebe" übertritt, anderseits aber auch darauf bezogen werden, dass infolge der Veränderung von $\mathrm{pH}$ des Blutes grobe Eiweissteilchen vom Eiweissreservoir in die Blutbahn in erheblichem Masse mobilisiert werden.

Bei Kaninchen, welche doppelseitiger Ureterenunterbindung unterworfen sind, macht die Säurezufuhr die mit der Urämie einhergehenden Azidosis an Grad noch stärker, was die Enniedrigung des Drucks pro \% bewirkt, indem die Bluteiweisskörper weiter nach grobdisperser Seite hin verschoben werden. Im Gegensatz hierzu nähert sich das $\mathrm{pH}$ des Blutes nach der Alkalizufuhr zur Norm und geht mit der Erhöhung des Drucks pro \% einher, was offenbar darauf zurückgeführt werden drüfte, dass sich der das Bluteiweissbild regulierende Mechanismus vorübergehend zur Norm erholt und die Zunahme 
der kleinen Eiweissteilchen im Blute bewirkt. Nach diesen bei urämischen Tieren erhobenen Ergebnissen lässt sich also sagen, dass, von kolloid-osmotischen Standpunkt betrachtet, bei geschädigter Nierenfunktion die Säurezufuhr schlechten Einfluss auf den Organismus ausübt, hingegen die Alkalizufuhr gewissermassen günstig wirkt.

\section{Schluss.}

1. Bei gesunden Kaninchen lässt die intravenöse Injektion von Säurebzw. Alkalilösung die Reaktion des Blutes vorübergehend nach saurer bzw. alkalischer Seite hin und zugleich die Bluteiweisskörper nach grobdisperser Seite hin verschieben.

2. Bei durch doppelseitige Ureterenunterbindung urämisch gewordenen Kaninchen erweist sich der Einfluss von Säure- bzw. Alkalizufuhr als folgendes: Die intravenöse Injektion von Säurelösung verschiebt die Reaktion des Blutes nach saurer Seite hin stärker und langdauernder, wodurch hervorgerufene Verschiebung der Bluteiweisskörper nach grobdisperser Seite hin an Grad stärker ist als bei gesunden Kaninchen. Bei intravenöser Verabreichung der Alkalilösung nähert sich die Reaktion des Blutes vorübergehend zur Norm, wobei das Bluteiweiss eine Verschiebung nach feindisperser Seite hin erleidet.

3. Betrachtet man die durch Säure- oder Alkalizufuht bedingten Veränderungen •des Bluteiweisses und dessen kolloid-osmotischen Drucks bei gestörter Nierenfunktion, so kann man schliessen, dass die Säurezufuhr, die eine augenfällige Erniedrigung des kolloid-osmotischen Drucks bewirkt, schlechten Einfluss auf das Krankheitsbild ausübt, wogegen die Alkalizufuhr vom kolloid-osmotischen Standpunkt beobachtet, günstig auf dasselbe einwirkt.

\section{Literatur.}

(1) Loeb, Arch. f. d. ges. Physiol., 1898, 69, 1; 1898, 71 , 457; 1899, 75, 303.

(2) Hamburger, Osmotischer Druck u. Ionenlehre, Wiesbaden 1902, 1, 327.

(3) Pfeiffer, Verh. d. deutsch. Kongr. f. inn. Med., 1911, 28, 506.

(4) Wyss, Deutsch. Arch. f. klin. Med., 1913, 111, 93.

(5) Maase u. Zondek, Das Hungerödem, Leipzig 1920.

(6) Jansen, Die Odemkrankheit, Leipzig 1920; zit. nach Zondek, Die Elektrolyte, Berlin 1927.

(7) Fischer, Oedema and Nephritis, New York 1915.

(8) Schade, Die physikalische Chemie, Dresden u. Leipzig 1920.

(9) Heymann, Arch. f. exp. Pathol. u. Pharm., 1921, 90, 27.

(10) Kuwabata, Fol. Pharm. Japon., 1928, 6, 440.

(11) Sato, Tohoku Journ. Exp. Med ; 1932, 18, 576.

(12) Völker, Zeitschr. f. d. ges. exp. Med., 1923, 37, 17.

(13) Gollwitzer-Meier u. Chr. Meyer, Ibid., 1924, 40, 70. 
(14) Kimura, Tohoku Journ. Exp. Med., 1930, 15, 199.

(15) Harkins u. Hastings, Journ. Biol, Chem., 1931, 90, 565.

(16) Laubender, Arch. f. exp. Path. u. Pharm., 1932, 165, 5.

(17) Kuge, Rinsyo Byorigaku Ketuekigaku Zassi, 1934. 3, 901.

(18) Oka, Tohoku Journ. Exp. Med., 1938, 32, 97.

(19) Ozawa, Aikawa u. A rakawa, Nippon Naikagakkai Zassi, 1921, 9, 407,

(20) Nisimura u. Takagaki, Osaka Ijisinsi, 1935, 6, 1178.

(21) Toth, Biochem. Ztschr., 1928, 201, 412.

(22) Elias u. Taubenhaus, Ztschr. f. ges. exp. Med., 1930, 74, 69.

(23) Loewy, Physiologie des Höhenklimas, Berlin 1932.

(24) Kasuga i, Tohoku Journ. Exp. Med., 1935, 27, 487.

(25) Sekiguti, Bulletin Naval Med. Assoc., 1936, 25, 634.

(26) Mi u ra, Tohoku Journ. Exp. Med., 1936, 30, 49.

(27) Is hikawa, Ibid., 1939, 36, 443.

(28) Gaza u. Brandi, Klin. Wschr., 1926, 1123.

(29) A u e rbach u. P ick, Arbeit. a. d. Reichsgesundheitsamte zu Berlin, 1912, 38, 256.

(30) Mis lowitzer, Die Bestimmung d. Wasserstoffionenkonzentration von Flüssigkeiten, Berlin 1928.

(31) Krogh u. Nakazawa, Biochem., Ztschr., 1927, 188, 241.

(32) Leendertz u. G tomelski, Arch. f. exp. Path. u. Pharm., 1922, 94, 114.

(33) Ito, Tohoku Journ. Exp. Med., 1929, 14; 198.

(34) Furuhasi, Aiti Igakkai Zassi, 1929, 36, 1707.

(35) Sasaki u. Yasuma, Ibid., 1933, 40, 1599.

(36) Onozaki, Tohoku Journ. Exp. Med. ;, 1935, 25, 1.

(37) Dienst, Arch. f. Gynäk., 1918, 109, 669.

(38) Kollert u. Starlinger, Ztschr. f. d. ges. exp. Med., 1922, 30, 293.

(39) Rusznyak, Barat u. Kürthy, Ztschr. f. klin. Med., 1924, 98, 337.

(40) Rusznyak, Ztschr. f. d. ges. exp. Med., 1924, 41, 532.

(41) Ozawa, Fukuoka Ikadaigaku Zassi, 1926, 19, 701.

(42) Tanaka, Ibid., 1928, 21, 948.

(43) Beckmann, Ztschr. f. d. ges. exp. Med., 1926, 51, 342.

(44) Bence, Ztschr. f. klin. Med., 1909, 67, 69.

(45) Litzner, Ibid., 1930, 112, 93.

(46) Kasiwamura, Zikken Syokakibyogaku, 1935, 10, 1041 u. 1149.

(47) Augelini, Rass. Ter. e Pat. Clin., 1934, 6, 257 ; Ref. in Kongresszbl. f. d. ges. inn. Med., 1934, 77, 429.

(48) Ok a, Tohoku Journ. Exp. Med., 1938, 32, 456. 